\title{
Photochemistry of Substituted Dibenzothiophene Oxides: The Effect of Trapping Groups
}

\author{
Supporting Information \\ Mrinmoy Nag and William S. Jenks* \\ Iowa State University \\ Ames, Iowa 50011-3111 \\ wsjenks@iastate.edu
}

\section{Table of Contents}

$\begin{array}{ll}\text { Experimental and synthetic details } & \text { S2 }\end{array}$

Spectral Data for compounds in numeric order S9 


\section{Experimental and synthetic details}

General. Reagents and solvents were used without further purification, except as noted. Dibenzothiophene was distilled under vacuum using a Kügelrohr apparatus at $175{ }^{\circ} \mathrm{C}$. All MS data were obtained in EI $(70 \mathrm{eV})$ or CI mode on a Finnigan TSQ 700 spectrometer. All NMR spectra were taken in $\mathrm{CDCl}_{3}$. Mass spectra were taken using EI mode, unless otherwise noted. 4Methyldibenzothiophene was prepared from DBT using a known procedure used by Katritzky. ${ }^{1}$

4-Vinyldibenzothiophene (4a), 4-allyldibenzothiophene (4b) and 4-Iododibenzothiophene ${ }^{2}$ (50 $\mathrm{mg}, 0.16 \mathrm{mmol})$ was dissolved in dry benzene $(3 \mathrm{~mL})$. To this was added tetrakis(triphenylphosphine)palladium $(18 \mathrm{mg}, 0.016 \mathrm{mmol})$ and vinyltributylstannane $(61.3 \mathrm{mg}$, $0.193 \mathrm{mmol})$ or allyltributylstannane $(60.9 \mathrm{mg}, 0.192 \mathrm{mmol})$. The reaction mixture was allowed to stir at reflux for about 24 hours under Ar. The reaction mixture was cooled and washed with saturated ammonium fluoride solution, followed by water and brine. The organic layer was evaporated and dried. The crude products were purified using preparatory TLC (hexane). The isolated yields of the 4-vinyl and 4-allyl derivatives were both about 55\%.

4a: ${ }^{1} \mathrm{H}$ NMR (400 MHz): $\delta 8.19-8.17(\mathrm{~m}, 2 \mathrm{H}), 8.1(\mathrm{~d}, 1 \mathrm{H}, \mathrm{J}=7.6 \mathrm{~Hz}), 7.92-7.88(\mathrm{~m}, 1 \mathrm{H}), 7.59$ $(\mathrm{d}, 1 \mathrm{H}, \mathrm{J}=7.6 \mathrm{~Hz}), 7.51-7.47(\mathrm{~m}, 1 \mathrm{H}), 7.03(\mathrm{dd}, 1 \mathrm{H}, \mathrm{J}=17.6 \mathrm{~Hz}, 11.2 \mathrm{~Hz}), 6.02(\mathrm{~d}, 1 \mathrm{H}, \mathrm{J}=17.6$ $\mathrm{Hz}), 5.57(\mathrm{~d}, 1 \mathrm{H}, \mathrm{J}=11.2 \mathrm{~Hz}) .{ }^{13} \mathrm{C}$ NMR (300 MHz): $\delta$ 145.0, 138.8, 136.4, 135.7, 135.1, 132.7, 127.0, 125.0, 124.68, 124.66, 122.9, 121.9, 121.0, 116.9. MS(EI) $\mathrm{m} / z$ 211, $210\left(\mathrm{M}^{+}\right), 209,183$.

4b: ${ }^{1} \mathrm{H}$ NMR (300 MHz): $\delta$ 8.18-8.13 (m, 1H), $8.06(\mathrm{~d}, 1 \mathrm{H}, \mathrm{J}=7.8 \mathrm{~Hz}), 7.90-7.86(\mathrm{~m}, 1 \mathrm{H})$, 7.50-7.45 (m, 2H), $7.45(\mathrm{t}, 1 \mathrm{H}, \mathrm{J}=7.2 \mathrm{~Hz}), 7.32(\mathrm{~d}, 1 \mathrm{H}, \mathrm{J}=7.5 \mathrm{~Hz}), 6.25-6.01(\mathrm{~m}, 1 \mathrm{H}), 5.23(\mathrm{dq}$, $1 \mathrm{H}, \mathrm{J}=1.5 \mathrm{~Hz}, 16.8 \mathrm{~Hz}), 5.19(\mathrm{dq}, 1 \mathrm{H}, \mathrm{J}=1.5 \mathrm{~Hz}, 9.9 \mathrm{~Hz}), 3.67(\mathrm{~d}, 2 \mathrm{H}, \mathrm{J}=6.3 \mathrm{~Hz}) \cdot{ }^{13} \mathrm{C}$ NMR (400 MHz): $\delta 146.1,144.5,143.5,140.1,135.1,134.3,126.8,126.6,125.1,124.6,123.0,121.9$, 119.8, 117.3, 39.6. MS(EI) m/z $224\left(\mathrm{M}^{+}\right), 208$. 
4-Homoallyldibenzothiophene (4c). To a solution of allylmagnesium bromide $(0.42 \mathrm{~mL}, 1 \mathrm{M}$ in ether, $0.42 \mathrm{mmol}$ ) held at about $0{ }^{\circ} \mathrm{C}$ was added a solution of 4-bromomethyldibenzothiophene $(5,57 \mathrm{mg}, 0.21 \mathrm{mmol})$ in $3 \mathrm{~mL}$ ether. The mixture was allowed to warm to room temperature and stirred for 2 days under argon. Saturated ammonium chloride solution was added to quench the reaction. Then it was washed with water and brine, dried over magnesium sulfate, and the solvent was removed. Purification was by prep TLC (hexane) to give $\mathbf{4 c}$ in $55 \%$ yield. ${ }^{1} \mathrm{H}$ NMR (400 MHz): $\delta 8.18-8.14(\mathrm{~m}, 1 \mathrm{H}), 8.04(\mathrm{~d}, 1 \mathrm{H}, \mathrm{J}=7.6 \mathrm{~Hz}), 7.91-7.88(\mathrm{~m}, 1 \mathrm{H}), 7.49-7.45(\mathrm{~m}, 2 \mathrm{H})$, $7.44(\mathrm{t}, 1 \mathrm{H}, \mathrm{J}=7.6 \mathrm{~Hz}), 7.31(\mathrm{~d}, 1 \mathrm{H}, \mathrm{J}=7.6 \mathrm{~Hz}) .5 .95(\mathrm{q}$ of $\mathrm{t}, 1 \mathrm{H}, \mathrm{J}=3.2 \mathrm{~Hz}, 10.4 \mathrm{~Hz}), 5.12$ (d of q, 1H, J = 1.6 Hz, 17.2 Hz), $5.02(\mathrm{dd}, 1 \mathrm{H}, \mathrm{J}=1.6 \mathrm{~Hz}, 10.4 \mathrm{~Hz}), 3.02(\mathrm{t}, 2 \mathrm{H}, \mathrm{J}=7.6 \mathrm{~Hz}), 2.59$ (q of $\mathrm{d}, 2 \mathrm{H}, \mathrm{J}=1.2 \mathrm{~Hz}, 8 \mathrm{~Hz}) .{ }^{13} \mathrm{C} \mathrm{NMR}(400 \mathrm{MHz}): \delta 13.9 .3,139.2,137.9,136.3,136.2,135.8$, 126.8, 126.4, 124.9, 124.5, 123.0, 121.9, 119.6, 115.5, 34.8, 33.3. MS(EI) m/z $238\left(\mathrm{M}^{+}\right), 197$.

General procedure for making DBTOs by oxidation of the corresponding sulfide. The sulfide (approximately $100 \mathrm{mg}$ ) was dissolved in dichloromethane (20 mL) and was cooled to $-30{ }^{\circ} \mathrm{C}$. To this solution, $m \mathrm{CPBA}(1 \mathrm{eq})$ in about $5 \mathrm{~mL}$ dichloromethane was added dropwise over a period of half an hour. After the addition, the mixture was allowed to stir for one hour at $-30{ }^{\circ} \mathrm{C}$, before being gradually warmed to room temperature. TLC was checked to monitor the reaction. The reaction stopped when the sulfone spot started showing up, which was generally about $1 \mathrm{~h}$ after addition was completed. To quench, the solution was washed with saturated sodium bicarbonate solution, and dried with $\mathrm{MgSO}_{4}$, following which the solvent was removed using a rotary evaporator to give a solid white product. The product was purified using preparatory TLC (1:1 hexane/ethyl acetate).

4-Vinyldibenzothiophene oxide (1a): Yield 40\%. ${ }^{1} \mathrm{H}$ NMR $(300 \mathrm{MHz}): \delta 7.96(\mathrm{~d}, 1 \mathrm{H}, \mathrm{J}=$ $7.5 \mathrm{~Hz}), 7.75(\mathrm{~d}, 1 \mathrm{H}, \mathrm{J}=7.5 \mathrm{~Hz}), 7.66(\mathrm{dd}, 1 \mathrm{H}, \mathrm{J}=1.2 \mathrm{~Hz}, 7.5 \mathrm{~Hz}), 7.60-7.45(\mathrm{~m}, 4 \mathrm{H})$, 
7.54(dd,1H), $7.39(\mathrm{dd}, 1 \mathrm{H}, \mathrm{J}=11.1 \mathrm{~Hz}, 17.4 \mathrm{~Hz}), 6.03(\mathrm{~d}, 1 \mathrm{H}, \mathrm{J}=17.4 \mathrm{~Hz}), 5.64(\mathrm{~d}, 1 \mathrm{H}, \mathrm{J}=11.1$ Hz). ${ }^{13} \mathrm{C}$ NMR (300 MHz): $\delta$ 144.6, 142.0, 138.6, 137.7, 133.0, 132.6, 131.8, 129.7, 127.5, 126.1, 122.1, 121.1, 119.5. MS(EI) $m / z 226\left(\mathrm{M}^{+}\right), 210$.

4-Allyldibenzothiophene oxide (1b): Yield 60\%. ${ }^{1} \mathrm{H}$ NMR (400MHz): $\delta 7.99$ (d of q, 1H, J = $0.4 \mathrm{~Hz}, 7.6 \mathrm{~Hz}), 7.80(\mathrm{~d}$ of q, 1H, J = 0.4 Hz, 7.6 Hz), $7.68(\mathrm{dd}, 1 \mathrm{H}, \mathrm{J}=1.2 \mathrm{~Hz}, 8.0 \mathrm{~Hz}), 7.60(\mathrm{td}$, $1 \mathrm{H}, \mathrm{J}=1.2 \mathrm{~Hz}, 7.6 \mathrm{~Hz}), 7.54(\mathrm{t}, 1 \mathrm{H}, \mathrm{J}=7.6 \mathrm{~Hz}), 7.50(\mathrm{td}, 1 \mathrm{H}, \mathrm{J}=1.2 \mathrm{~Hz}, 7.6 \mathrm{~Hz}), 7.29(\mathrm{dd}, 1 \mathrm{H}$, $\mathrm{J}=0.4 \mathrm{~Hz}, 7.6 \mathrm{~Hz}), 6.13-6.03(\mathrm{~m} .1 \mathrm{H}), 5.36(\mathrm{dq}, 1 \mathrm{H}, \mathrm{J}=1.6 \mathrm{~Hz}, 17.6 \mathrm{~Hz}), 5.21(\mathrm{dq}, 1 \mathrm{H}, 1.2 \mathrm{~Hz}$, $10.0 \mathrm{~Hz}), 3.98(\mathrm{dd}, 1 \mathrm{H}, \mathrm{J}=7.2 \mathrm{~Hz}, 15.6 \mathrm{~Hz}), 3.81(\mathrm{dd}, 1 \mathrm{H}, \mathrm{J}=6.4 \mathrm{~Hz}, 15.6 \mathrm{~Hz}) .{ }^{13} \mathrm{C}$ NMR $(400$ MHz): $\delta 144.9,143.2,141.6,137.7,137.4,135.2,133.1,132.6,130.4,129.7,127.6,122.1$, 120.1, 117.8, 37.0. MS(EI) $m / z, 240\left(\mathbf{M}^{+}\right), 224 / 223$.

4-(3-butenyl)dibenzothiophene oxide (1c): Yield 45\%. ${ }^{1} \mathrm{H}$ NMR (300 MHz): $\delta 7.99$ (d of q, $1 \mathrm{H}, \mathrm{J}=0.6 \mathrm{~Hz}, 6.9 \mathrm{~Hz}), 7.80(\mathrm{dd}, 1 \mathrm{H}, \mathrm{J}=0.6 \mathrm{~Hz}, 7.8 \mathrm{~Hz}), 7.66(\mathrm{dd}, 1 \mathrm{H}, \mathrm{J}=0.6 \mathrm{~Hz}, 7.5 \mathrm{~Hz}), 7.6$ $(\mathrm{t}$ of d, $1 \mathrm{H}, \mathrm{J}=1.2 \mathrm{~Hz}, 7.5 \mathrm{~Hz}), 7.53(\mathrm{t}, 1 \mathrm{H} . \mathrm{J}=7.5 \mathrm{~Hz}), 7.50(\mathrm{t}$ of d, $1 \mathrm{H}, \mathrm{J}=7.5 \mathrm{~Hz}), 7.29(\mathrm{~d}, 1 \mathrm{H}$, $\mathrm{J}=7.5 \mathrm{~Hz}), 5.93(\mathrm{q}$ of $\mathrm{t}, 1 \mathrm{H}, \mathrm{J}=6.6 \mathrm{~Hz}, 10.2 \mathrm{~Hz}), 5.11(\mathrm{~m}, 1 \mathrm{H}), 5.04(\mathrm{~m}, 1 \mathrm{H}), 3.21(\mathrm{~m}, 2 \mathrm{H}), 2.61$ (m, 2H). ${ }^{13} \mathrm{C}$ NMR (400 MHz): $\delta$ 140.9, 138.3, 138.1, 133.9, 133.8, 132.1, 131.8, 131.4, 130.4, 122.2, 121.6, 119.5, 119.2, 115.4, 33.6, 30.9. MS(EI) $\mathrm{m} / z 254\left(\mathrm{M}^{+}\right), 237$.

4-Fluorodibenzothiophene oxide (6). 4-Fluorodibenzothiophene was first prepared by adaptation of a literature method. ${ }^{3}$ To a cold $\left(-50{ }^{\circ} \mathrm{C}\right)$ solution of DBT $(0.40 \mathrm{~g}, 2.17 \mathrm{mmol})$ in dry THF ( $8 \mathrm{~mL})$ was added N-butyllithium $(1.2 \mathrm{~mL}, 1.4 \mathrm{eq})$. The solution was allowed to warm up to room temperature and stirred for $6 \mathrm{~h}$. The solution was then cooled to $-40{ }^{\circ} \mathrm{C}$, and $\mathrm{N}$ fluorobenzenesulfonimide $(0.69 \mathrm{~g}, 2 \mathrm{eq})$ in dry THF $(10 \mathrm{~mL})$ was added to the reaction pot. The solution was allowed to warm up to the room temperature and was stirred for another 16 hours. The reaction mixture was treated with saturated ammonium chloride solution and then washed 
with water and brine. The organic layer was dried with $\mathrm{MgSO}_{4}$, and solvent was removed using a rotary evaporator to give solid mixture of compounds. The compound was purified by column chromatography (hexane/silica). ${ }^{1} \mathrm{H}$ NMR (300 MHz): $\delta 8.16-8.13(\mathrm{~m}, 1 \mathrm{H}), 7.93(\mathrm{dd}, 1 \mathrm{H}, \mathrm{J}=0.6$ Hz, 8.1 Hz), 7.91-7.88 (m, 1H), 7.54-7.47 (m, 2H), $7.43(\mathrm{td}, 1 \mathrm{H}, \mathrm{J}=4.8 \mathrm{~Hz}, 8.1 \mathrm{~Hz}), 7.20$ (td, J $0.9 \mathrm{~Hz}, 8.1 \mathrm{~Hz}) .{ }^{13} \mathrm{C}$ NMR (300 MHz): $\delta 159.6(\mathrm{~d}, \mathrm{~J}=79.8 \mathrm{~Hz}), 139.7(\mathrm{~d}, \mathrm{~J}=4.2 \mathrm{~Hz}), 139.1$ (d, J $=18.9 \mathrm{~Hz}), 135.4(\mathrm{~d}, \mathrm{~J}=9.0 \mathrm{~Hz}), 127.5,126.0(\mathrm{~d}, \mathrm{~J}=27 \mathrm{~Hz}), 124.9,123.2,122.2,117.4(\mathrm{~d}, \mathrm{~J}=$ $13.5 \mathrm{~Hz}), 112.4,112$.

The oxidation of the product to the sulfoxide $\mathbf{6}$ was done without further purification by the general procedure outlined previously using $m$-CPBA. The product was purified using preparatory TLC (1:1 hexane/ethyl acetate). ${ }^{1} \mathrm{H}$ NMR $(300 \mathrm{MHz}): \delta 7.99(1 \mathrm{H}, \mathrm{J}=0.6 \mathrm{~Hz}, 7.5$ Hz), $7.80(\mathrm{dd}, 1 \mathrm{H}, \mathrm{J}=0.6 \mathrm{~Hz}, 7.2 \mathrm{~Hz}), 7.64-7.58(\mathrm{~m}, 3 \mathrm{H}), 7.54(\mathrm{td}, 1 \mathrm{H}, \mathrm{J}=1.2 \mathrm{~Hz}, 4.5 \mathrm{~Hz}), 7.19-$ $7.13(\mathrm{~m}, 1 \mathrm{H}) .{ }^{13} \mathrm{C}$ NMR $(300 \mathrm{MHz}): \delta 163.7,160.3,145.6,140.6(\mathrm{~d}, \mathrm{~J}=13.2 \mathrm{~Hz}), 136.6(\mathrm{~d}, \mathrm{~J}=$ 9.6 Hz), $135.6(\mathrm{~d}, \mathrm{~J}=30.9 \mathrm{~Hz}), 132.9,130.4,127.8,122.6,117.9(\mathrm{~d}, \mathrm{~J}=13.8 \mathrm{~Hz}), 116.8(\mathrm{~d}, \mathrm{~J}=$ $79.5 \mathrm{~Hz})$.

4-Bromomethyldibenzothiophene oxide. 4-Bromomethyldibenzothiophene was prepared from 4-methyldibenzothiophene using a known procedure of Kudo. ${ }^{4}$ The oxidation to the sulfoxide was done without further purification by the general procedure outlined previously using $m$-CPBA. $\quad{ }^{1} \mathrm{H}$ NMR (400 MHz): $\delta 8.01(\mathrm{~d}, 1 \mathrm{H}, \mathrm{J}=7.6 \mathrm{~Hz}), 7.81(\mathrm{~d}, 1 \mathrm{H}, \mathrm{J}=8.0 \mathrm{~Hz}), 7.76$ $(\mathrm{d}, 1 \mathrm{H}, \mathrm{J}=7.6 \mathrm{~Hz}), 7.62(\mathrm{td}, 1 \mathrm{H}, \mathrm{J}=1.2 \mathrm{~Hz}, 7.6 \mathrm{~Hz}), 7.59(\mathrm{t}, 1 \mathrm{H}, \mathrm{J}=7.6 \mathrm{~Hz}), 7.53(\mathrm{td}, 1 \mathrm{H}, \mathrm{J}=$ $1.2 \mathrm{~Hz}, 7.6 \mathrm{~Hz}), 7.48(\mathrm{~d}, 1 \mathrm{H}, \mathrm{J}=7.6 \mathrm{~Hz}), 4.93(\mathrm{dd}, 2 \mathrm{H}, \mathrm{J}=10.8 \mathrm{~Hz}, 218 \mathrm{~Hz}) .{ }^{13} \mathrm{C} \mathrm{NMR}(400$ MHz): $\delta 144.7,143.3,138.8,138.1,136.8,133.5,132.8,131.0,130.0,127.7,122.3,122.1,28.0$. 
(7b). 4-Fluorodibenzothiophene oxide, 4-bromomethyldibenzothiophene oxide, 4fluorodibenzothiophene or 4-bromomethyldibenzothiophene, as appropriate, was dissolved in anhydrous DMF (5 mL/1 mg, approximately $50 \mathrm{mg}$ scale). Sodium thiomethoxide (3 eq), dissolved in about $10 \mathrm{~mL}$ DMF, was added to the solution and the temperature was raised to 90 ${ }^{\circ} \mathrm{C}$ for one day. The reaction mixture was quenched with sodium bicarbonate and extracted with chloroform. The organic layer was washed with water until neutral (pH paper) and dried over magnesium sulfate. The solvent was removed and the product was purified using preparatory TLC using 1:1 hexane, ethyl acetate mixture as the eluent.

2a: Yield 65\%. ${ }^{1} \mathrm{H}$ NMR (300 MHz): $\delta 8.00(\mathrm{dq}, 1 \mathrm{H}, \mathrm{J}=0.6 \mathrm{~Hz}, 7.5 \mathrm{~Hz}), 7.79(\mathrm{dq}, 1 \mathrm{H}, \mathrm{J}=0.6$ $\mathrm{Hz}, 7.5 \mathrm{~Hz}), 7.61(\mathrm{tt}, 2 \mathrm{H}, \mathrm{J}=1.2 \mathrm{~Hz}, 7.8 \mathrm{~Hz}), 7.56(\mathrm{t}, 1 \mathrm{H}, \mathrm{J}=7.8 \mathrm{~Hz}), 7.52(\mathrm{td}, 1 \mathrm{H}, \mathrm{J}=1.2 \mathrm{~Hz}$, $7.5 \mathrm{~Hz}), 7.36(\mathrm{dd}, 1 \mathrm{H}, \mathrm{J}=1.2 \mathrm{~Hz}, 7.8 \mathrm{~Hz}), 2.65$ (s, 3H). ${ }^{13} \mathrm{C}$ NMR (400 MHz): $\delta$ 145.1, 143.2, 141.1, 138.2, 137.0, 133.4, 132.6, 130.0, 127.6, 127.5, 122.3, 118.9. MS(EI) $m / z 246\left(\mathrm{M}^{+}\right), 229$, 216.

2b: Yield 55\%. ${ }^{1} \mathrm{H}$ NMR (300 MHz): $\delta 7.98(\mathrm{dq}, 1 \mathrm{H}, \mathrm{J}=0.6 \mathrm{~Hz}, 7.5 \mathrm{~Hz}), 7.81(\mathrm{dq}, 1 \mathrm{H}, \mathrm{J}=$ $0.6 \mathrm{~Hz}, 7.5 \mathrm{~Hz}), 7.71(\mathrm{dd}, 1 \mathrm{H}, \mathrm{J}=1.2 \mathrm{~Hz}, 7.5 \mathrm{~Hz}), 7.60(\mathrm{td}, 1 \mathrm{H}, \mathrm{J}=1.2 \mathrm{~Hz}, 7.5 \mathrm{~Hz}), 7.56(\mathrm{t}, 1 \mathrm{H}$, $\mathrm{J}=7.5 \mathrm{~Hz}), 7.51(\mathrm{td}, 1 \mathrm{H}, \mathrm{J}=1.2 \mathrm{~Hz}, 7.5 \mathrm{~Hz}), 7.45(\mathrm{~d}, 1 \mathrm{H}, \mathrm{J}=7.2 \mathrm{~Hz}), 4.18(\mathrm{dd}, 2 \mathrm{H}, \mathrm{J}=14.1 \mathrm{~Hz}$ $24.0 \mathrm{~Hz}$ ), 2.10 (s, 3H). ${ }^{13} \mathrm{C}$ NMR (400 MHz): $\delta$ 140.4, 138.9, 136.7, 135.9, 133.4, 128.8, 127.5, 125.4, 125.1, 123.1, 122.2, 122.0, 38.1, 15.6. MS(EI) $m / z 244\left(\mathrm{M}-16^{+}\right), 243,227,197 . \mathrm{MS}(\mathrm{Ion}$ Trap) $m / z, 261\left(\mathrm{M}+1^{+}\right), 244,227,197$.

7a: Yield 65\%. ${ }^{1} \mathrm{H}$ NMR (300 MHz): $\delta$ 8.16-8.13 (m, 1H), $8.02(\mathrm{dd}, 1 \mathrm{H}, \mathrm{J}=1.8 \mathrm{~Hz}, 6.9 \mathrm{~Hz})$, 7.93-7.89 (m, 1H), 7.50-7.42 (m, 4H), 2.54 (s, 3H). MS(EI) m/z $230\left(\mathrm{M}^{+}\right), 215,184,171$. Data matched reported spectra from alternate preparative route. ${ }^{5}$ 
7b: Yield 60\%. H NMR (300 MHz): $\delta$ 8.18-8.15 (m, 1H), $8.11(\mathrm{td}, 1 \mathrm{H}, \mathrm{J}=1.2 \mathrm{~Hz}, 7.5 \mathrm{~Hz})$, 7.91-7.89 (m, 1H), 7.51-7.43 (m, 3H), $7.40(\mathrm{dd}, 1 \mathrm{H}, \mathrm{J}=1.2 \mathrm{~Hz}, 7.5 \mathrm{~Hz}) \cdot{ }^{13} \mathrm{C}$ NMR $(400 \mathrm{MHz}): \delta$ 133.5, 130.7, 128.2, 128.0, 127.5, 126.3, 125.3, 124.8, 124.4, 123.0, 121.9, 121.7, 29.9, 12.0. MS(EI) $m / z 244 \quad\left(\mathrm{M}^{+}\right), \quad 197,184 . \quad$ The immediate precursor of $\mathbf{7 b}$ was (4-bromomethyl)dibenzothiophene, which was not completely purified. The reaction mixture of 7b contained a small quantity of the starting material and 4-methyldibenzothiophene. All three compounds has very similar chromatographic behavior; hence $\mathbf{7 b}$ could not be purified completely. But the proton-NMR spectrum shows $\mathbf{7 b}$ is the major component in the mixture, and GC-MS analysis also demonstrates the major peak is $\mathbf{7 b}$. Because this was a potential product, rather than a starting material for photochemistry, we did not pursue quantitative purification.

4-methylsulfinyldibenzothiophene (11a) Compound 11a was made by the oxidation of 7a using the same general procedure used for making other DBTOs. One equivalent $m$-CPBA could only oxidize the aryl-alkyl sulfide leaving the ring sulfur intact. The compound was purified using preparatory TLC (1:1 methylene chloride/ethyl acetate). Yield 70\%. ${ }^{1} \mathrm{H}$ NMR (300 MHz): $\delta 8.43(\mathrm{dd}, 1 \mathrm{H}, \mathrm{J}=1.2 \mathrm{~Hz}, 7.8 \mathrm{~Hz}), 8.24-8.21(\mathrm{~m}, 1 \mathrm{H}), 8.12(\mathrm{dd}, 1 \mathrm{H}, \mathrm{J}=1.2 \mathrm{~Hz}, 7.8$ $\mathrm{Hz}), 7.95-7.92(\mathrm{~m}, 1 \mathrm{H}), 7.67(\mathrm{t}, 1 \mathrm{H}, \mathrm{J}=7.8 \mathrm{~Hz}), 7.58-7.54(\mathrm{~m}, 2 \mathrm{H}), 3.19(\mathrm{~s}, 3 \mathrm{H}) .{ }^{13} \mathrm{C}$ NMR $(300$ MHz): $\delta 140.3,138.3,138.0,134.4,134.2,128.2,127.5,126.7,125.3,125.1,122.9,122.1,43.1$. MS(EI) $m / z 246\left(\mathbf{M}^{+}\right), 231,182$.

\section{4-(2-Propylthioethyl)dibenzothiophene oxide (2c) and 4-(3-propylthiopropyl)-}

dibenzothiophene oxide (2d) 4-Vinyldibenzothiophene oxide (62 mg, $0.27 \mathrm{mmol}$ ) or 4-allyl dibenzothiophene oxide (23 mg, $0.096 \mathrm{mmol})$, AIBN (10 mol\%), and propanethiol (3.6 eq) were dissolved in $10 \mathrm{~mL} \mathrm{CCl}_{4}$. The reaction mixture was refluxed under Argon for one day. Then the 
reaction mixture was washed with $1 \mathrm{~N}$ sodium hydroxide, followed by several portions of water until the solution became neutral. Then the extract was dried over anhydrous magnesium sulfate. Purification by preparatory TLC (1:1 hexane/ethyl acetate) gave pure $\mathbf{2 c}$ (50 $\mathrm{mg}, 60 \%)$ or $\mathbf{2 d}$ (13 $\mathrm{mg}, 43 \%)$.

2c: ${ }^{1} \mathrm{H}$ NMR $(300 \mathrm{MHz}): \delta 7.98(\mathrm{~d}, 1 \mathrm{H}, \mathrm{J}=7.8 \mathrm{~Hz}), 7.79(\mathrm{~d}, 1 \mathrm{H}, \mathrm{J}=7.5 \mathrm{~Hz}), 7.54(\mathrm{t}, 1 \mathrm{H}, \mathrm{J}=$ $7.8 \mathrm{~Hz}), 7.50(\mathrm{td}, 1 \mathrm{H}, \mathrm{J}=1.2 \mathrm{~Hz}, 7.5 \mathrm{~Hz}), 7.32(\mathrm{~d}, 1 \mathrm{H}, \mathrm{J}=7.5 \mathrm{~Hz}), 3.37$ (t, 2H, J = 7.5 Hz), 3.09$2.86(\mathrm{~m}, 2 \mathrm{H}), 2.61(\mathrm{t}, 2 \mathrm{H}, \mathrm{J}=7.5 \mathrm{~Hz}), 1.66(\operatorname{sextet}, 2 \mathrm{H}, \mathrm{J}=7.5 \mathrm{~Hz}), 1.01(\mathrm{t}, 3 \mathrm{H}, \mathrm{J}=7.5 \mathrm{~Hz}) .{ }^{13} \mathrm{C}$ NMR (300 MHz): $\delta 144.8,143.6,142.2,137.7,137.5,133.1,132.7,130.7,129.7,127.5,122.1$, 120.3, 34.5, 33.9, 33.1, 23.1, 13.7. $\mathrm{MS}(\mathrm{EI}) \mathrm{m} / z .302\left(\mathrm{M}^{+}\right), 285,251,243$.

2d: ${ }^{1} \mathrm{H}$ NMR (300 MHz): $\delta 7.98(\mathrm{dd}, 1 \mathrm{H}, \mathrm{J}=0.6 \mathrm{~Hz}, 7.2 \mathrm{~Hz}), 7.80(\mathrm{dd}, 1 \mathrm{H}, \mathrm{J}=0.6 \mathrm{~Hz}, 7.5$ Hz), $7.67(\mathrm{dd}, 1 \mathrm{H}, \mathrm{J}=1.2 \mathrm{~Hz}, 7.5 \mathrm{~Hz}), 7.60(\mathrm{dt}, 1 \mathrm{H}, \mathrm{J}=1.2 \mathrm{~Hz}, 7.5 \mathrm{~Hz}), 7.53(\mathrm{t}, 1 \mathrm{H}, \mathrm{J}=7.5 \mathrm{~Hz})$, $7.50(\mathrm{dt}, 1 \mathrm{H}, \mathrm{J}=1.2 \mathrm{~Hz}, 7.5 \mathrm{~Hz}), 7.30(\mathrm{~d}, 1 \mathrm{H}, \mathrm{J}=7.2 \mathrm{~Hz}), 3.33-3.10(\mathrm{~m}, 2 \mathrm{H}), 2.64(\mathrm{t}, 2 \mathrm{H}, \mathrm{J}=7.5$ Hz), $2.54(\mathrm{t}, 2 \mathrm{H}, \mathrm{J}=7.5 \mathrm{~Hz}), 2.19-2.09(\mathrm{~m}, 2 \mathrm{H}), 1.63$ (septet, 2H, J = 7.5 Hz), 1.00 (t, 3H, J = 7.5 Hz). ${ }^{13} \mathrm{C}$ NMR (400 MHz): $\delta 149.5,145.9,143.4,137.5,135.0,133.1,132.6,130.3,129.7$, 127.6, 122.1, 119.9, 34.3, 32.2, 31.7, 30.9, 23.2, 13.8. MS(EI) $m / z 316\left(\mathrm{M}^{+}\right), 223$. 


\section{Spectra}

\section{4-Vinyldibenzothiophene oxide (1a)}

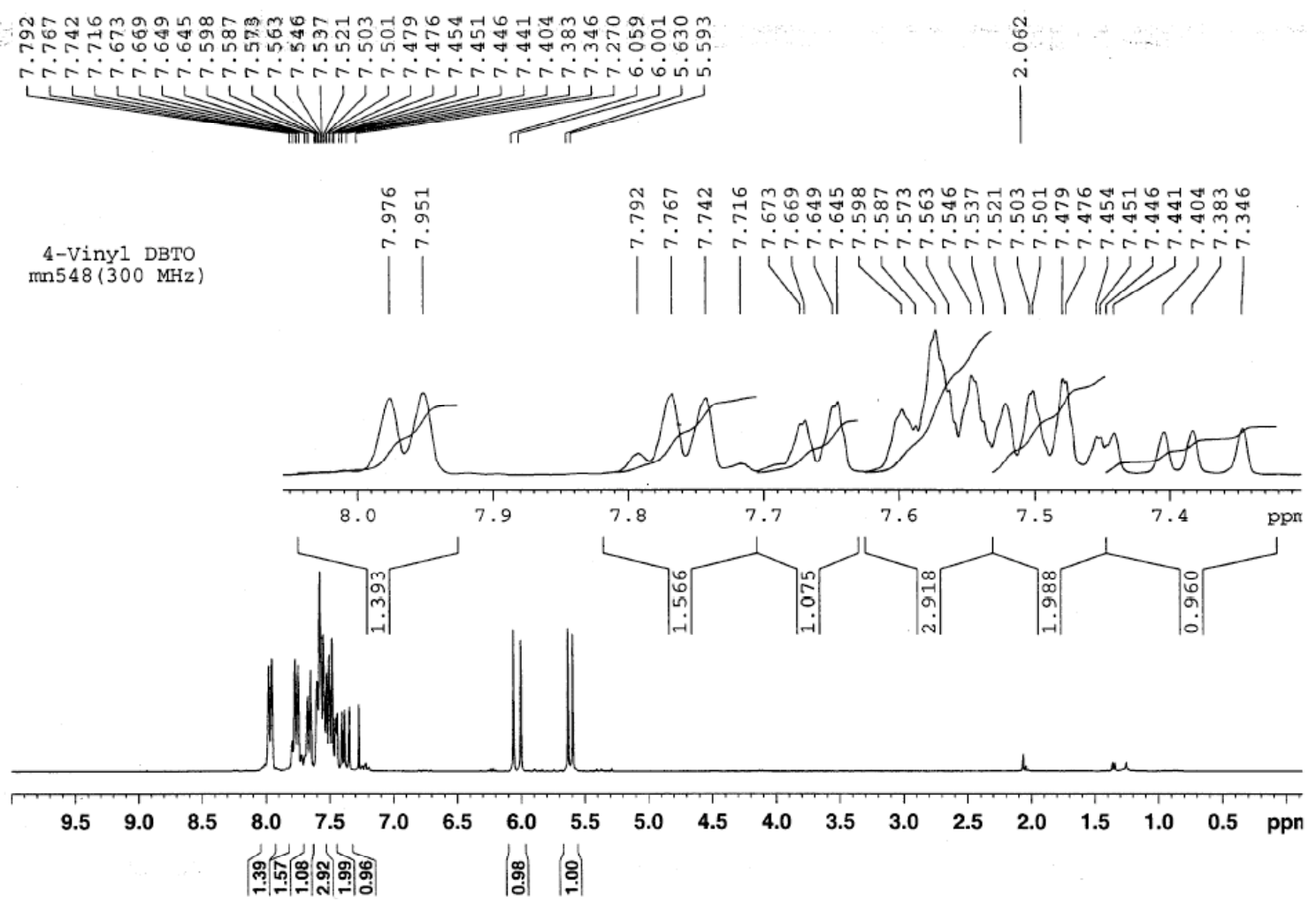



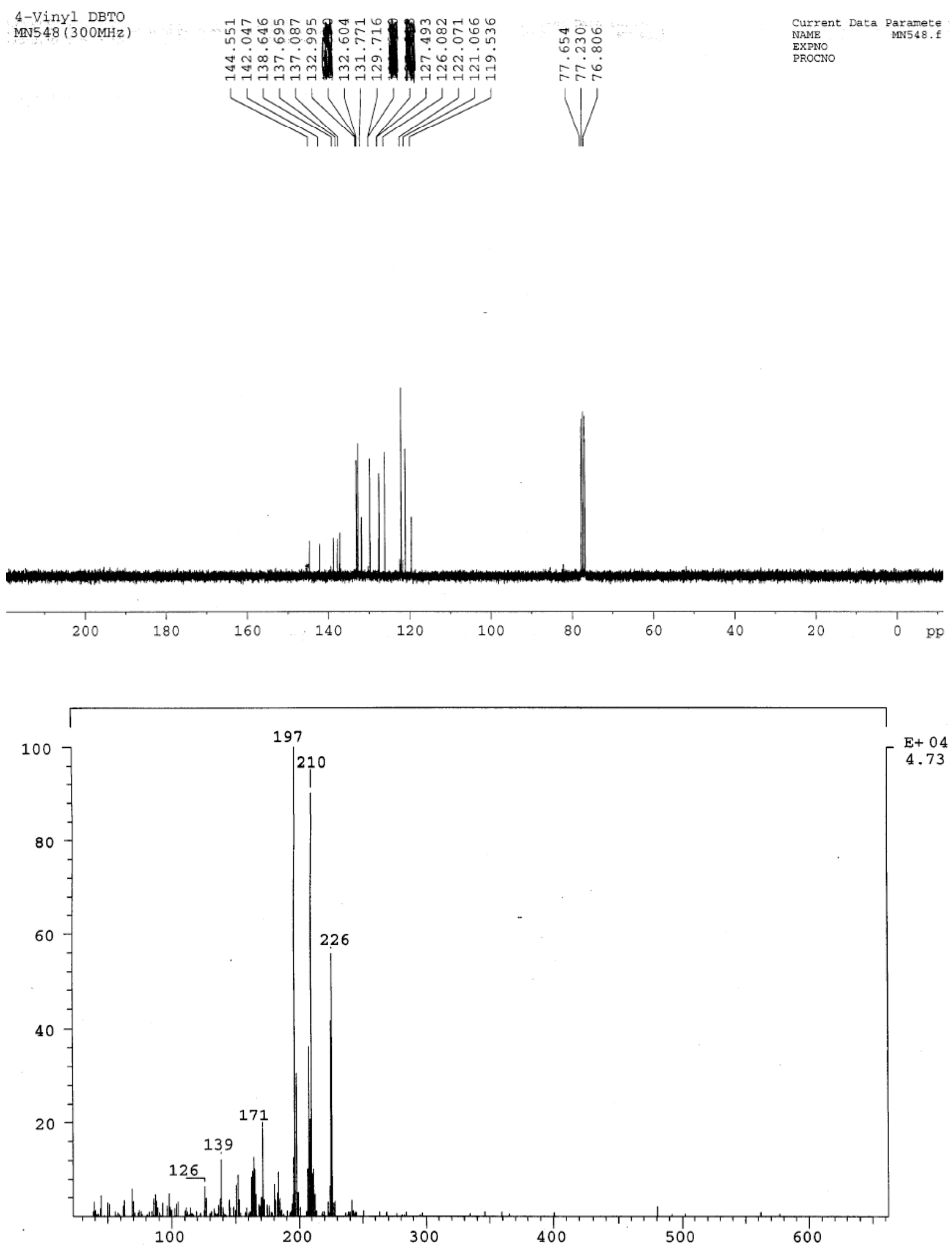


\section{4-Allyldibenzothiophene oxide (1b)}
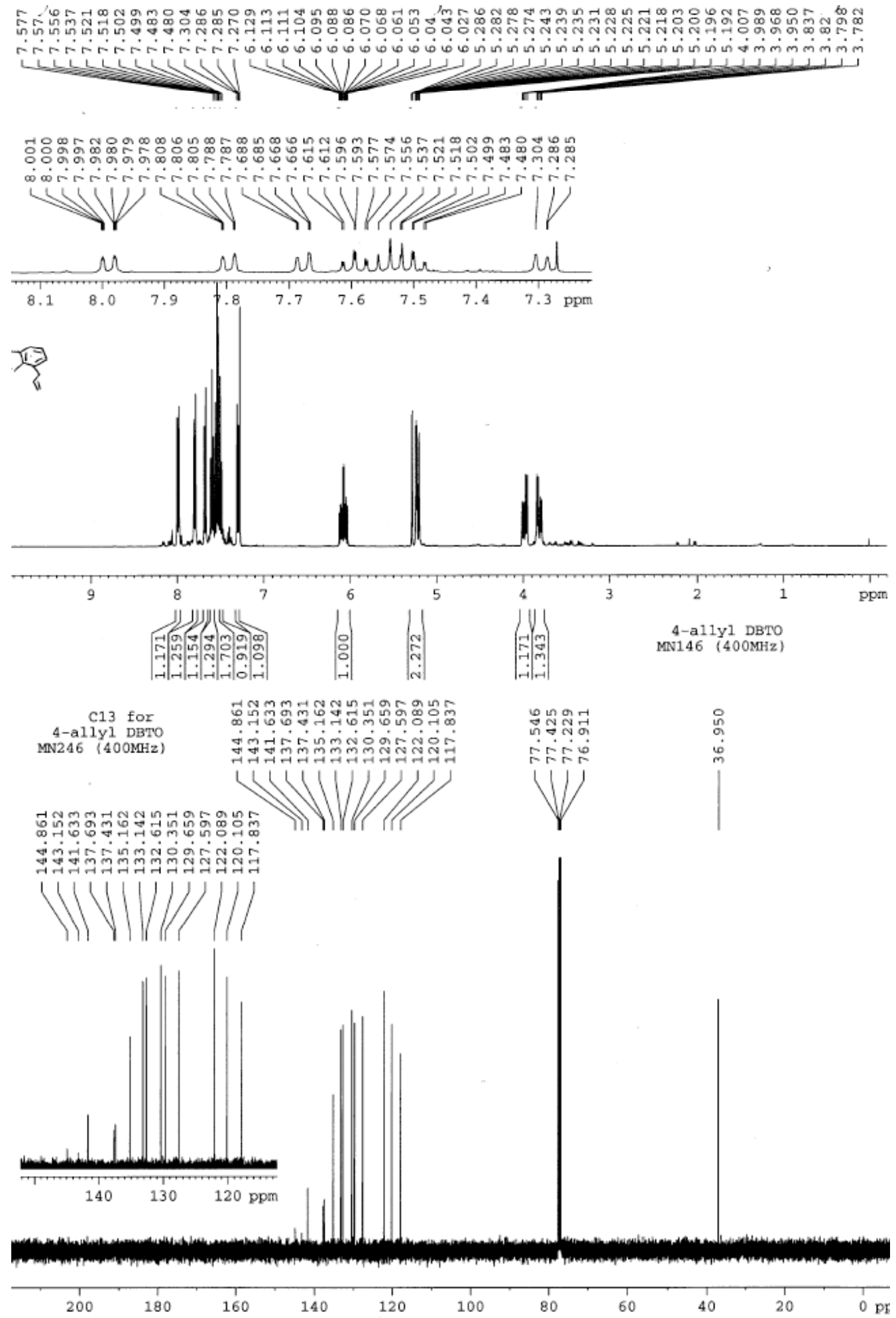


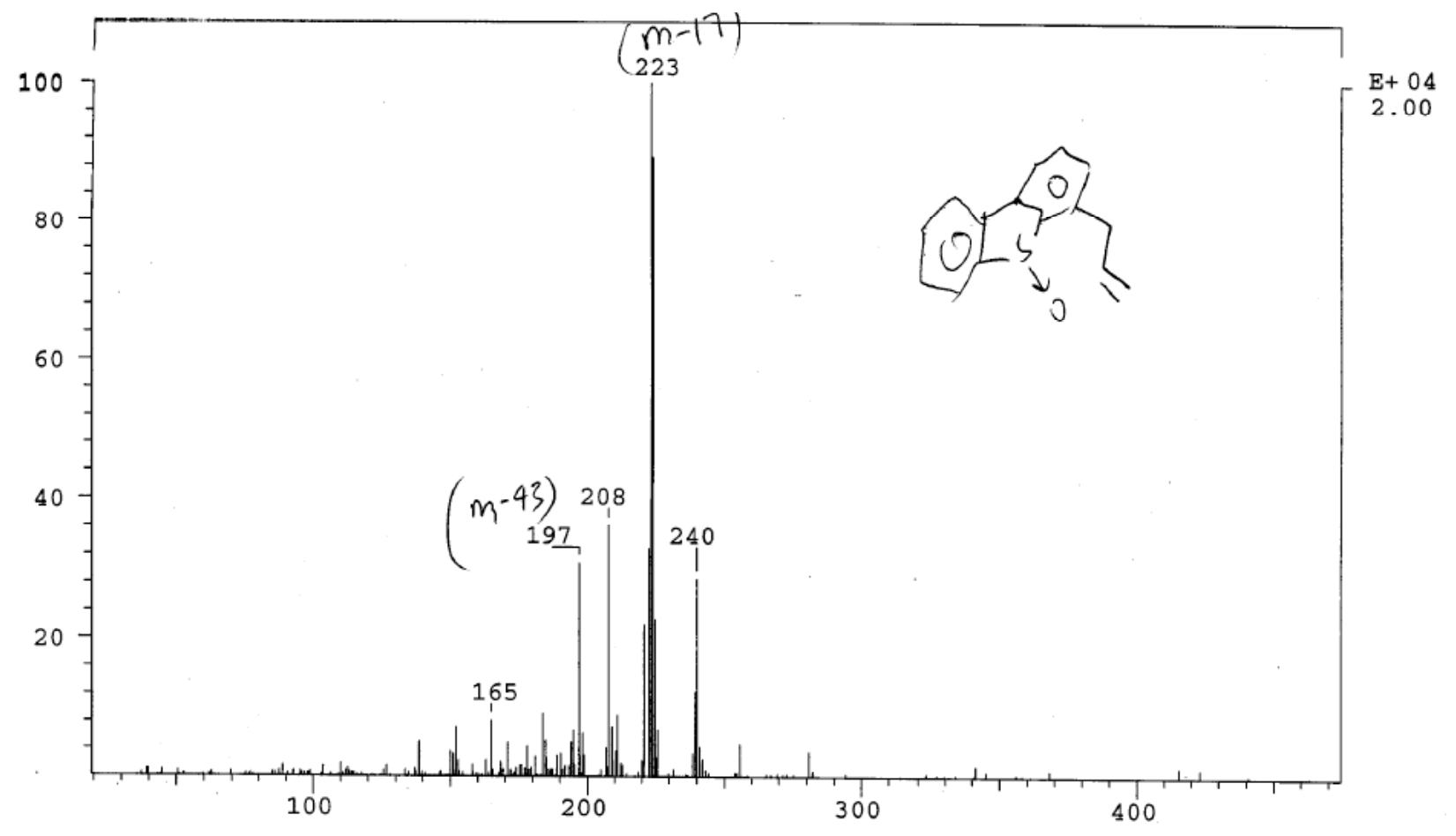




\section{4-(3-butenyl)dibenzothiophene oxide (1c)}

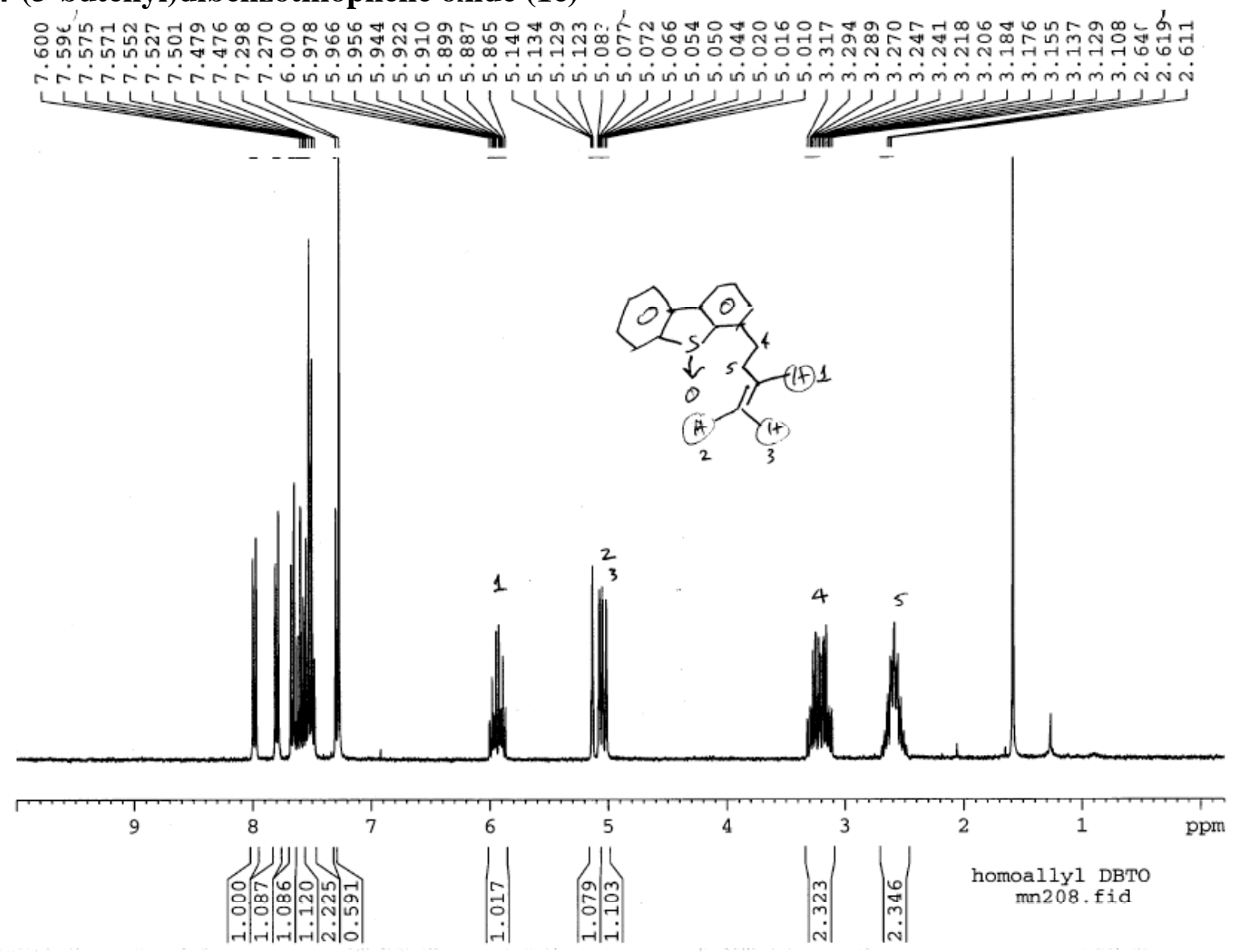

aboting

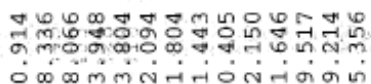

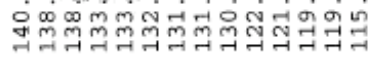

4-Homoally1 DBTO

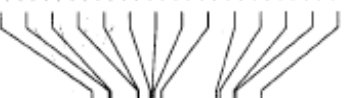

MN563 (DRX 400)
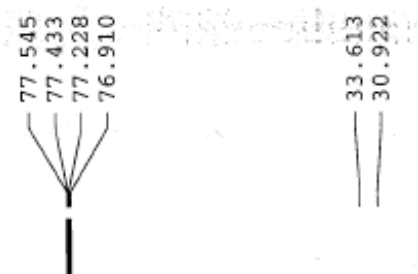

(a)

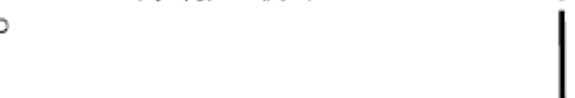




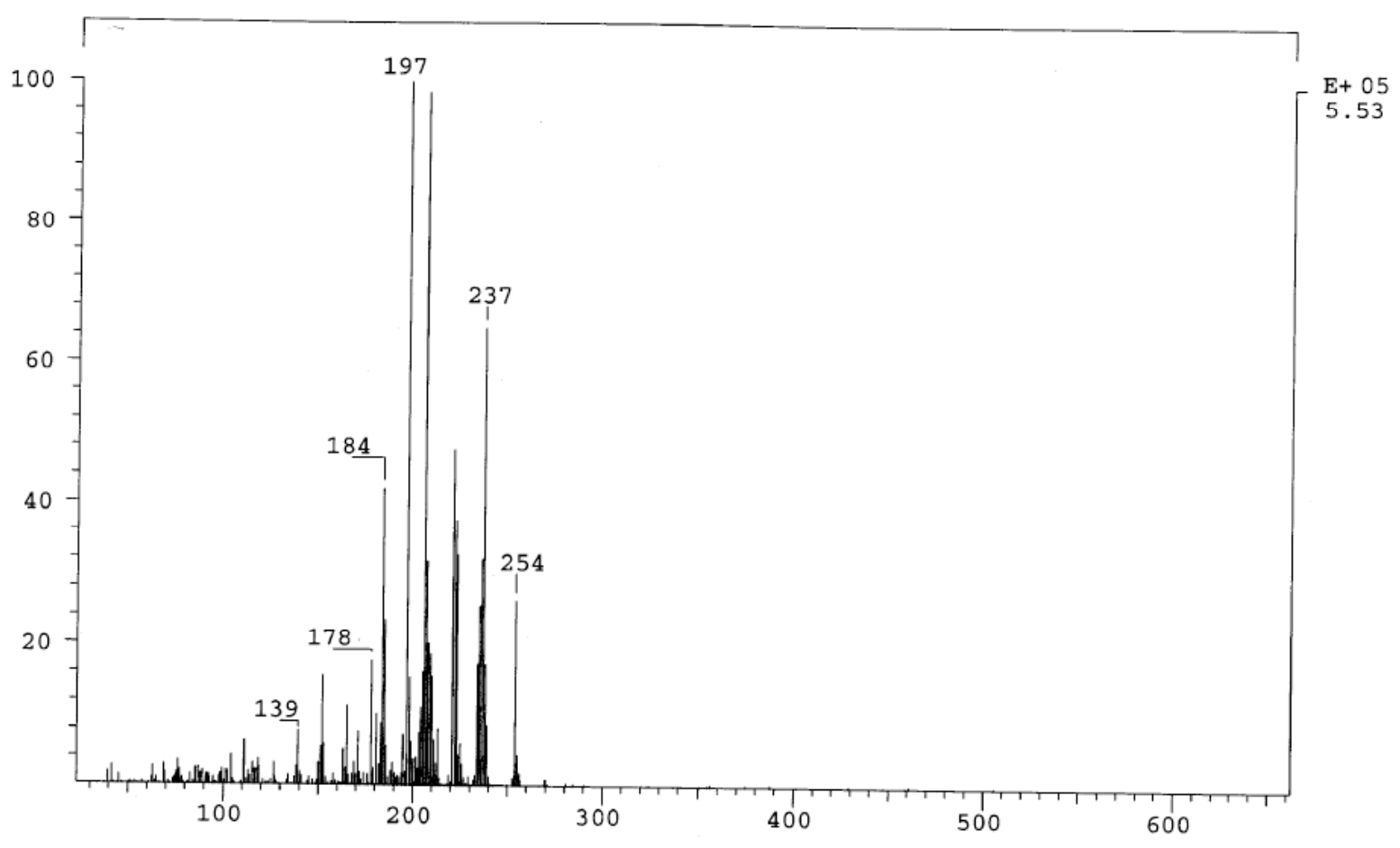




\section{4-Methylthiodibenzothiophene oxide (2a)}

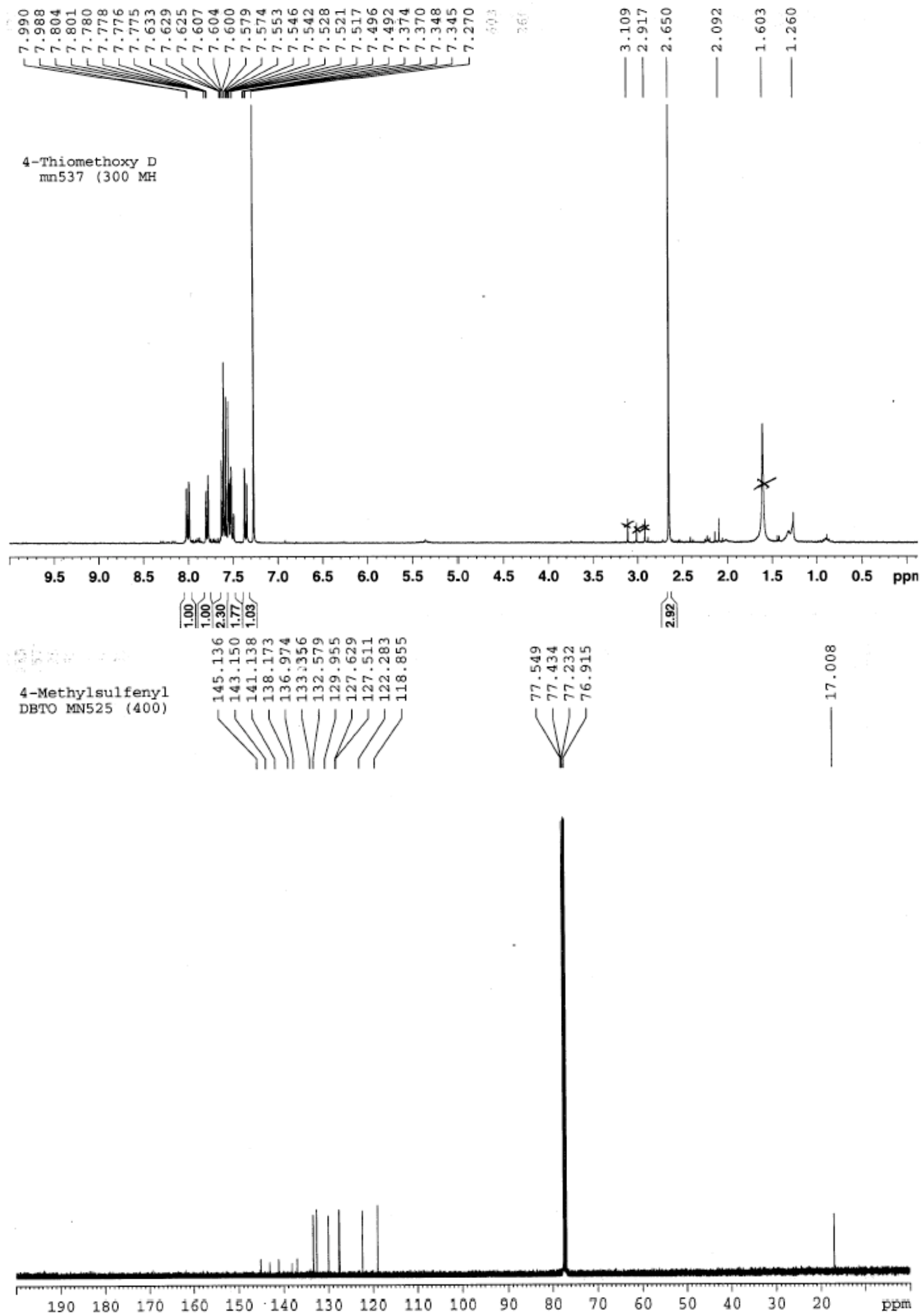




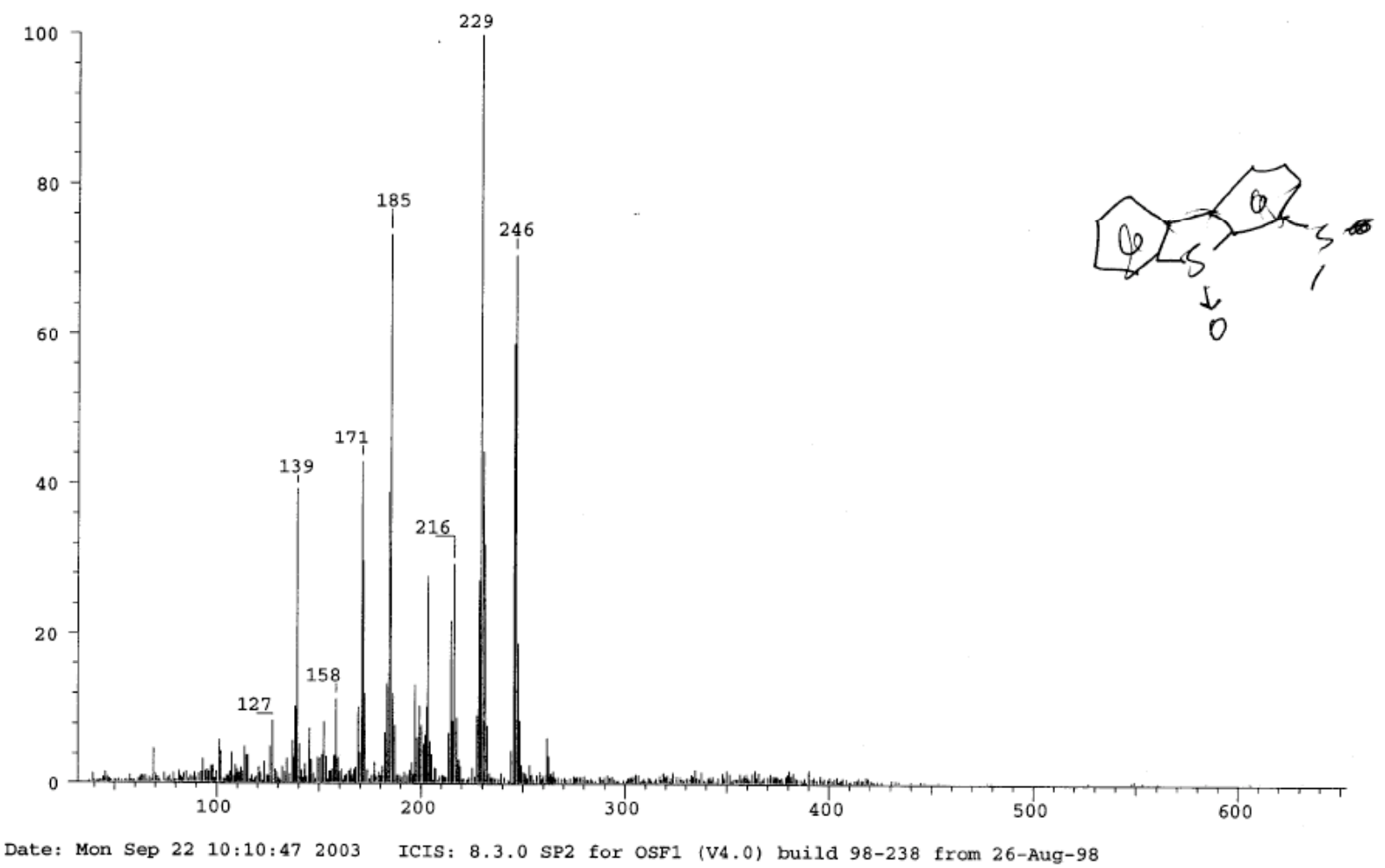


4-Methylthiomethyldibenzothiophene oxide (2b)
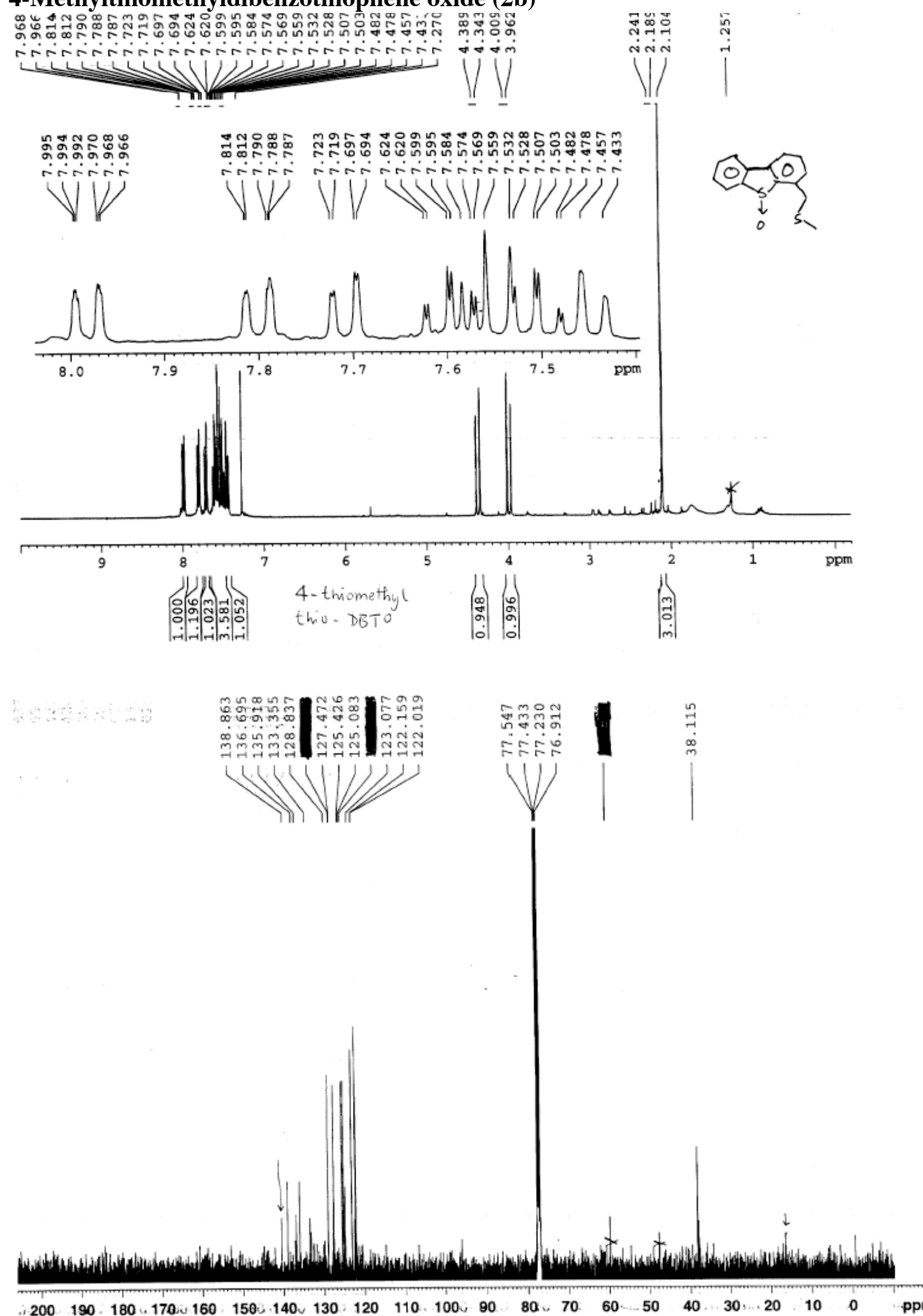

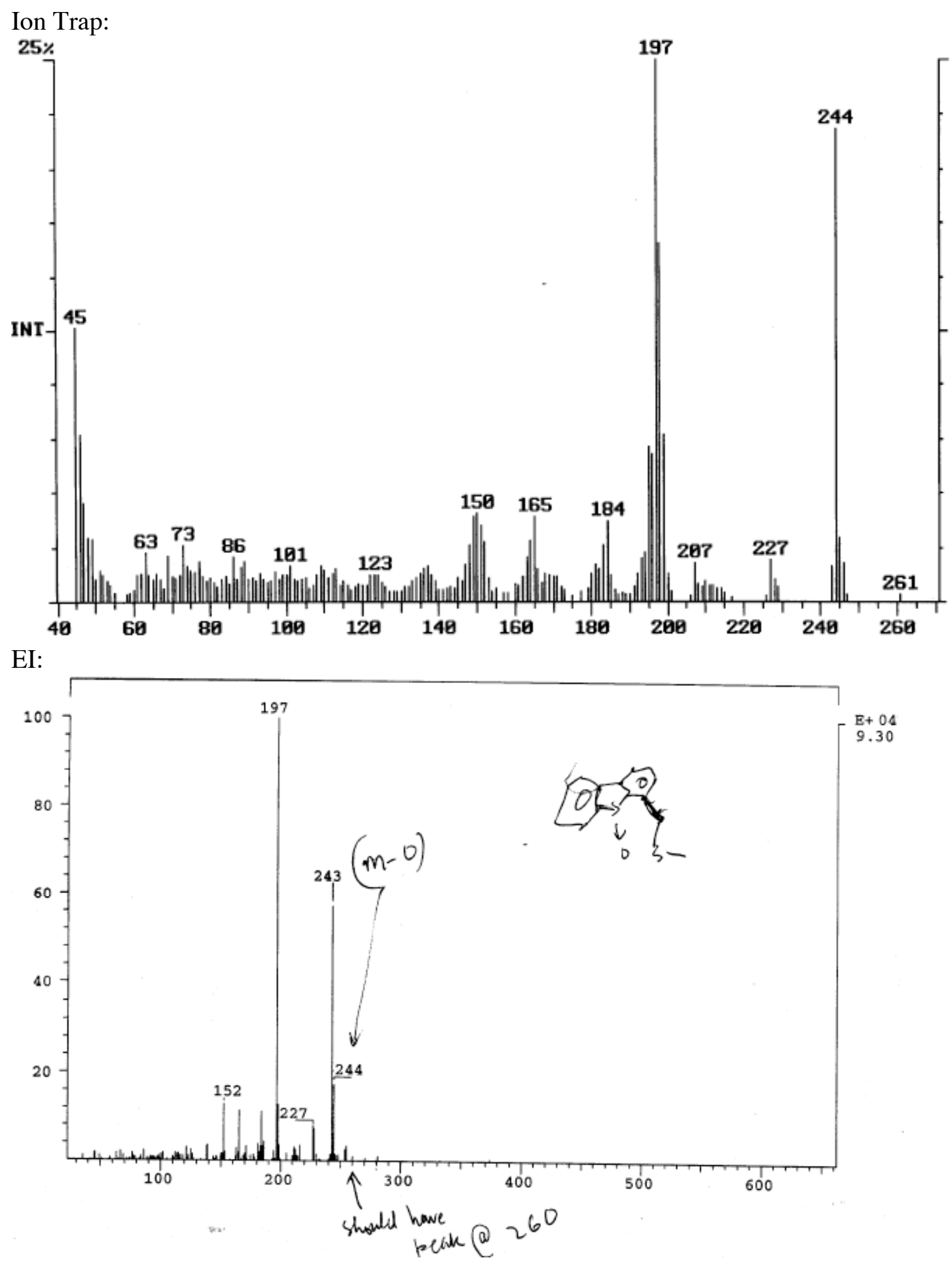


\section{4-(2-Propylthioethyl)dibenzothiophene oxide (2c)}

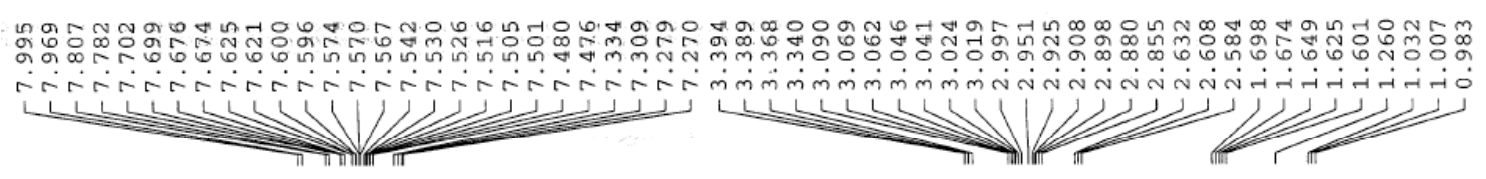

4-(2-sulfanyl-propy 1$)$

-ethyl DBTO mn555

$300 \mathrm{MHz}$

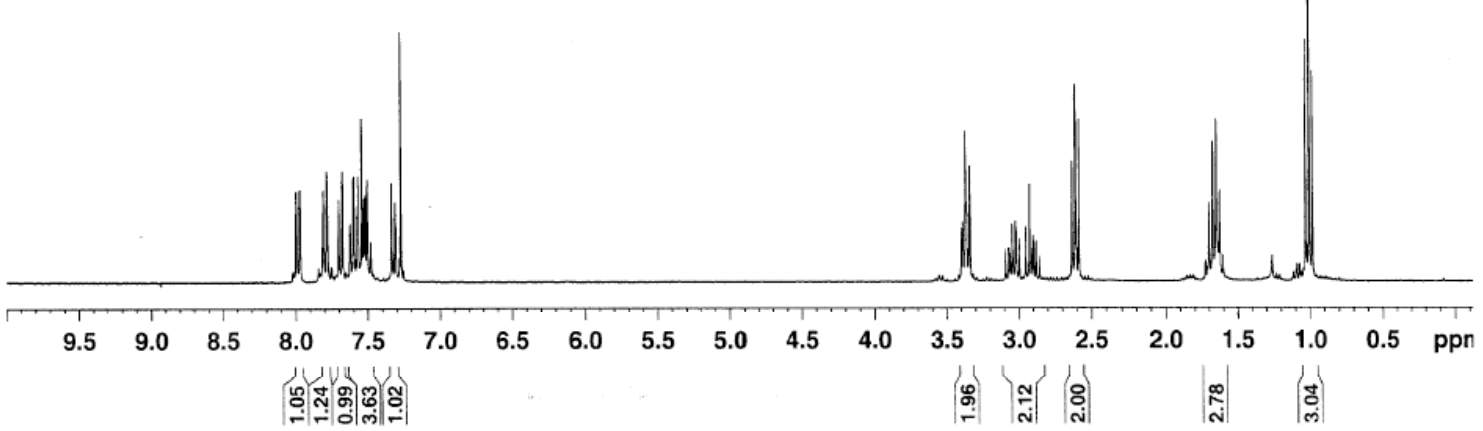

4-(2-sulfanylpropy $)$
-ethyl DBTO MN555

$300 \mathrm{MHz}$
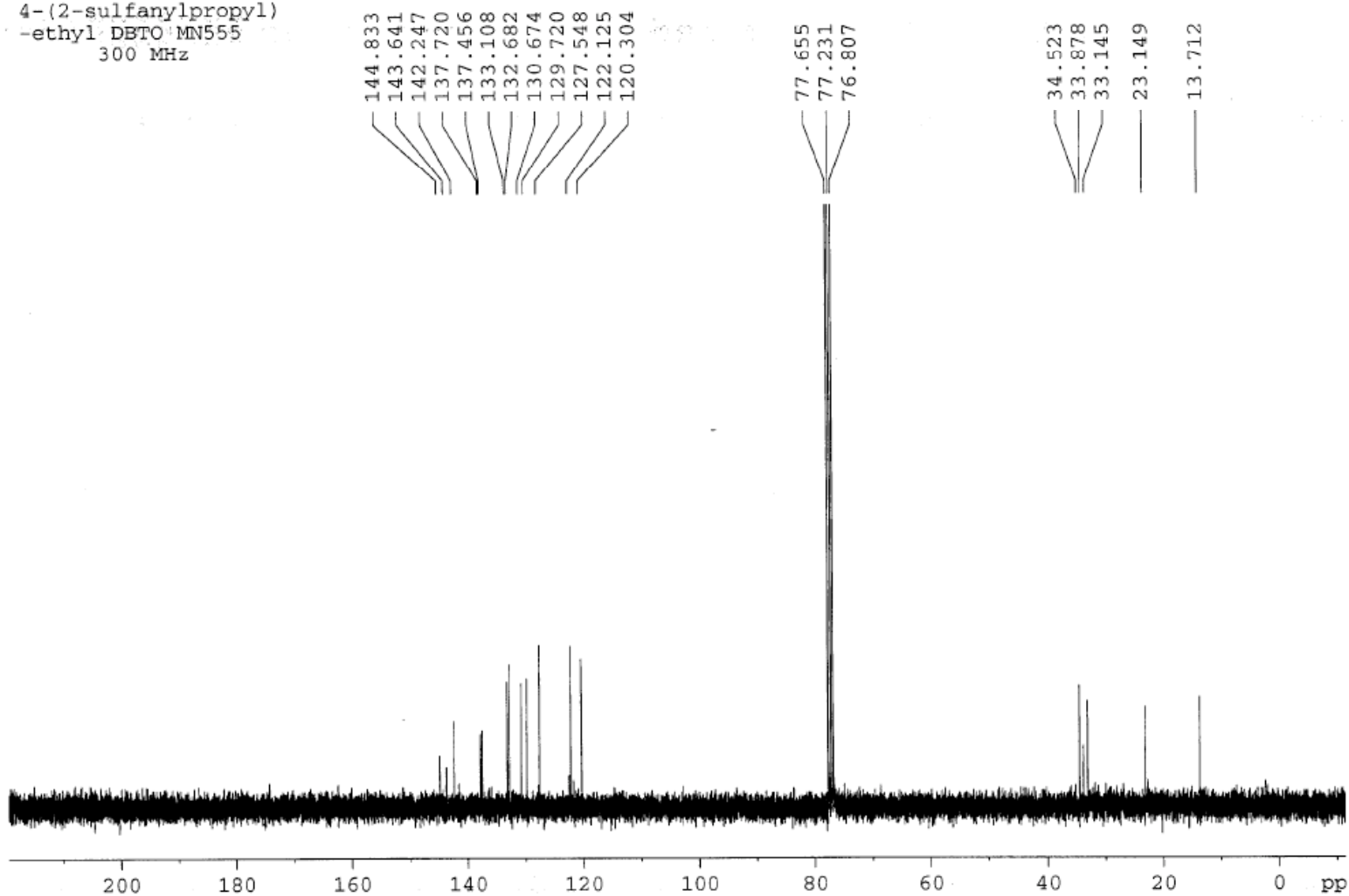


\section{4-(3-Propylthiopropyl)dibenzothiophene oxide (2d)}

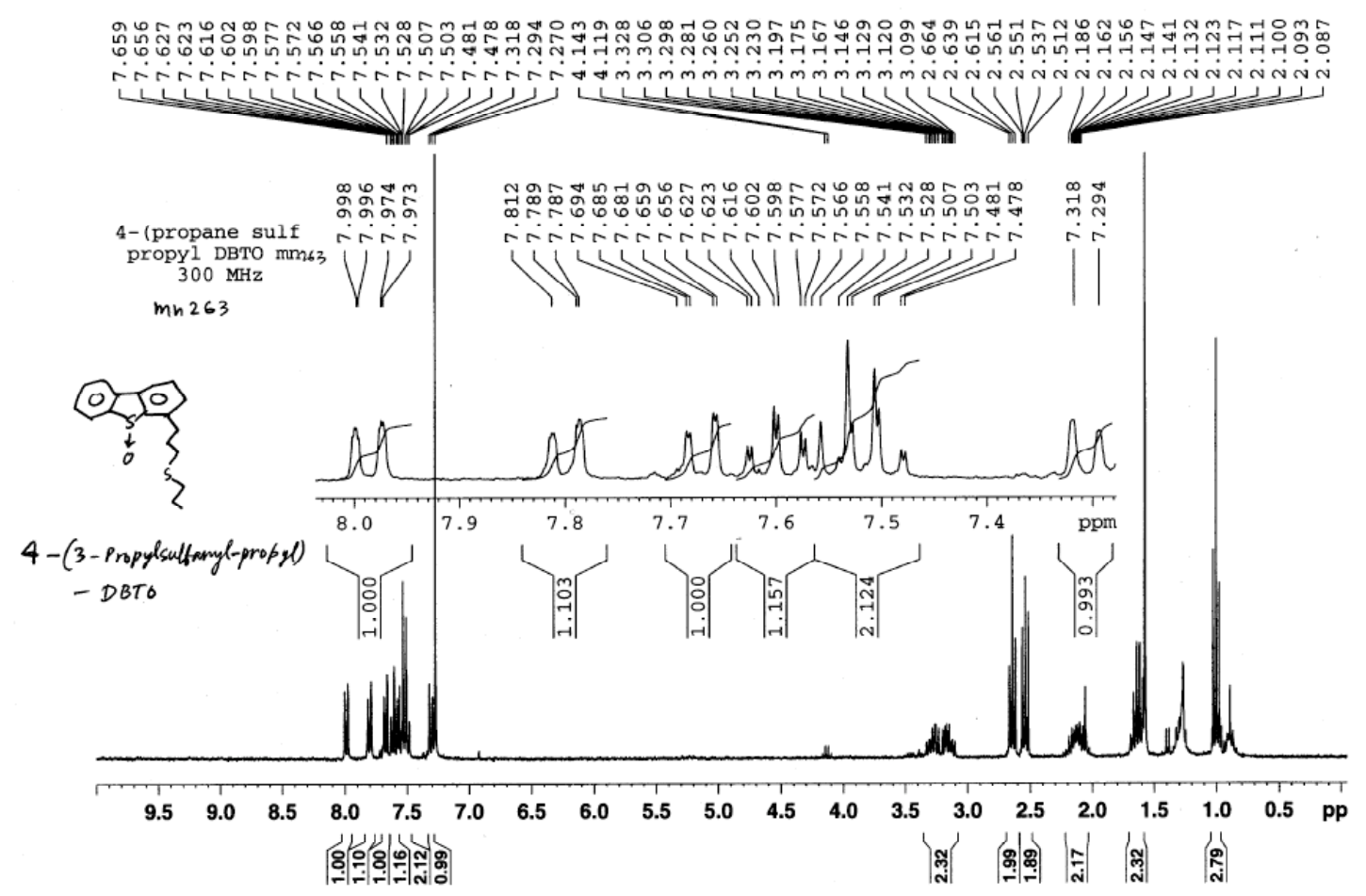

4-(3-1, isulfanyl-propyl) -

$D B T O$

4-(proparesulfeny I)

p 1 DBTO MNC251

$02,>0$

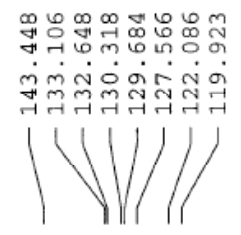

$\begin{array}{ll}\infty & 0 \\ 0 & 0\end{array}$

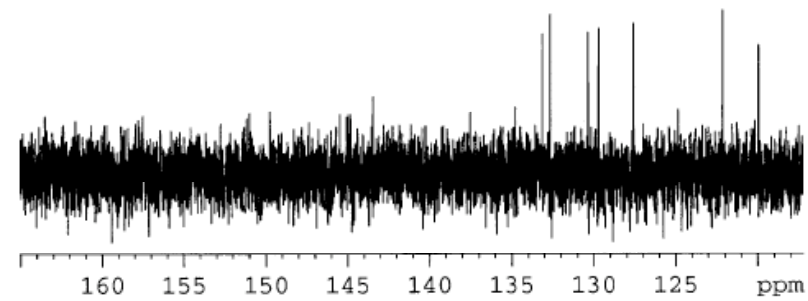

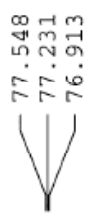

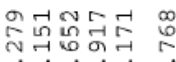

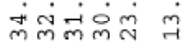

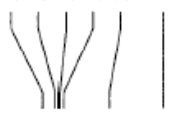

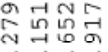

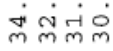
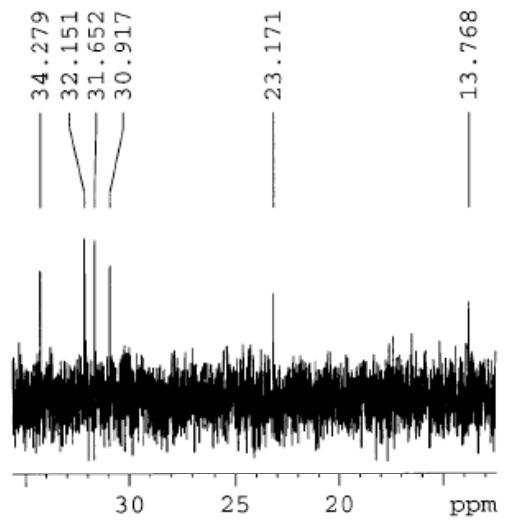


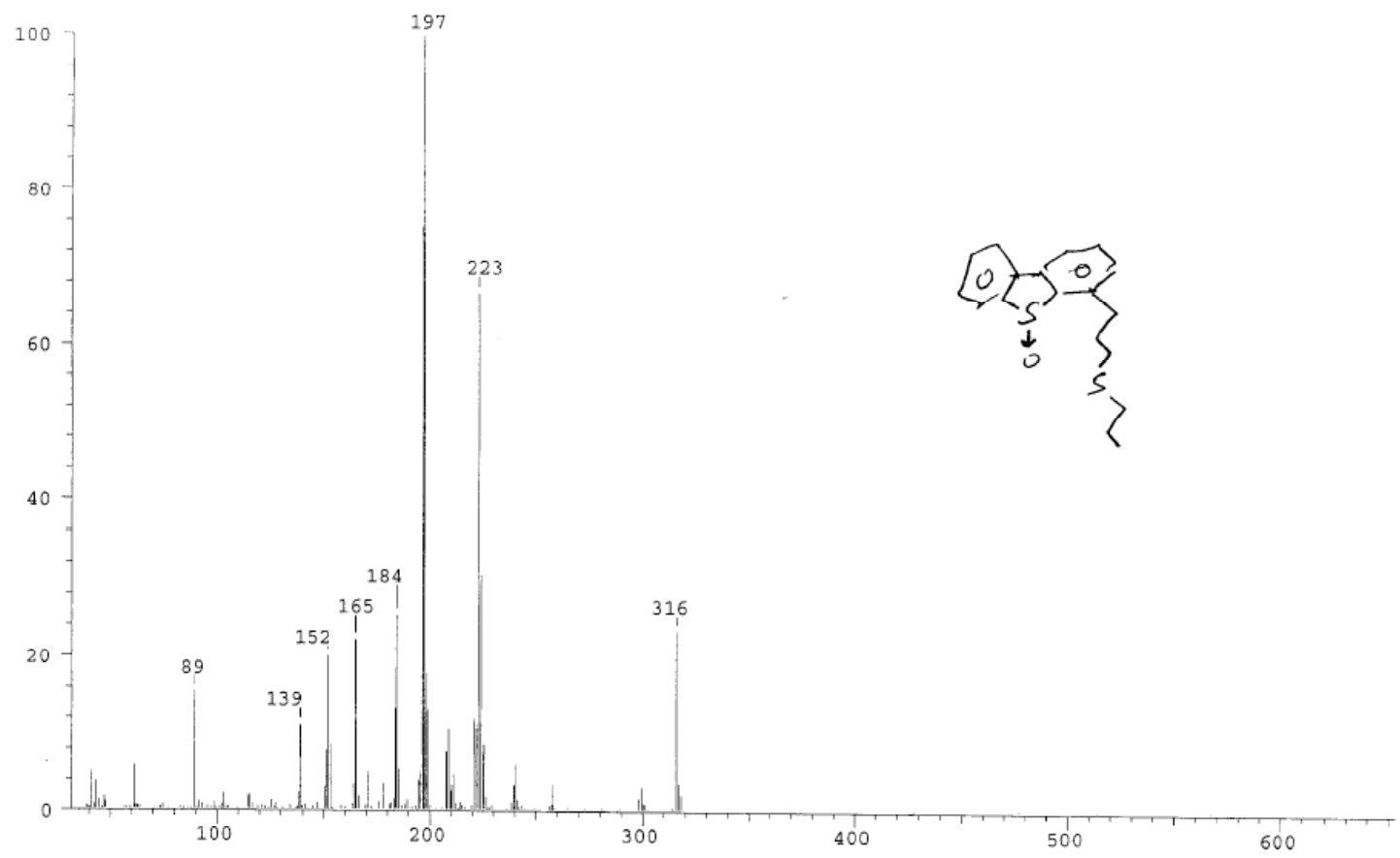

Date: Mon Sep 29 11:48:41 2003

ICIS: 8.3.0 SP2 for OSF1 (V4.0) build 98-238 from 26-Aug-98 


\section{4-Vinyldibenzothiophene (4a)}
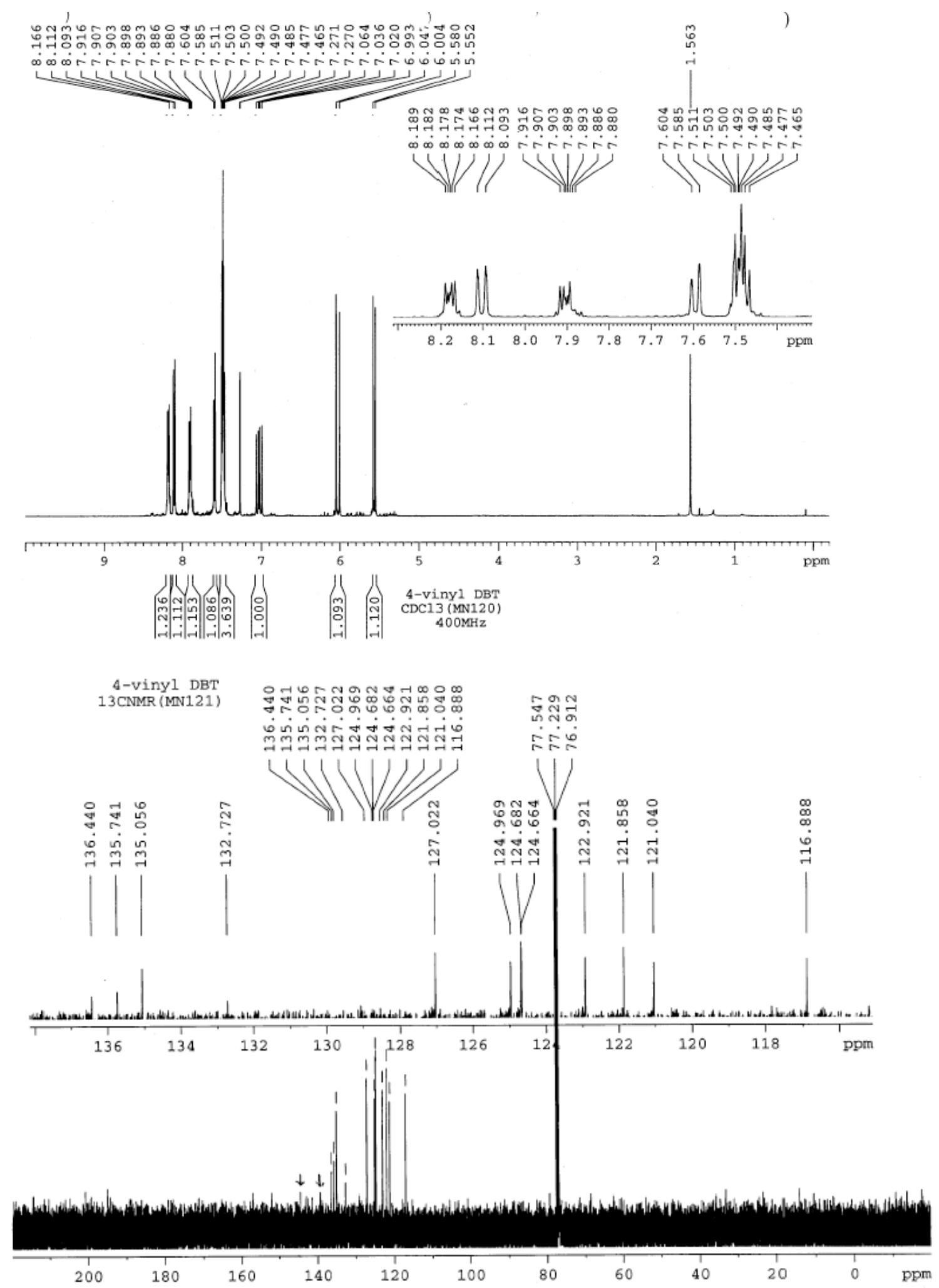


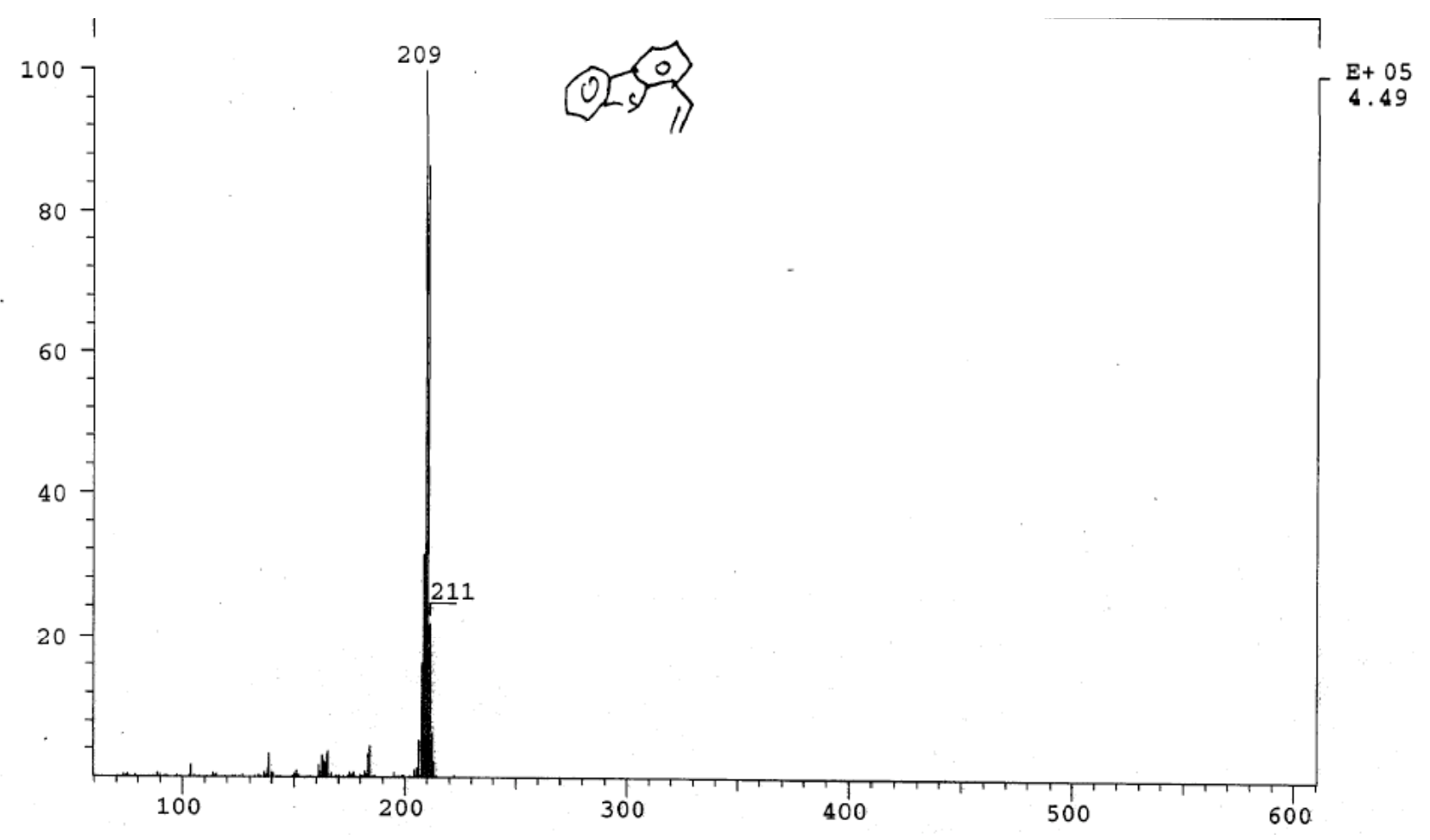


4-Allyldibenzothiophene (4b)
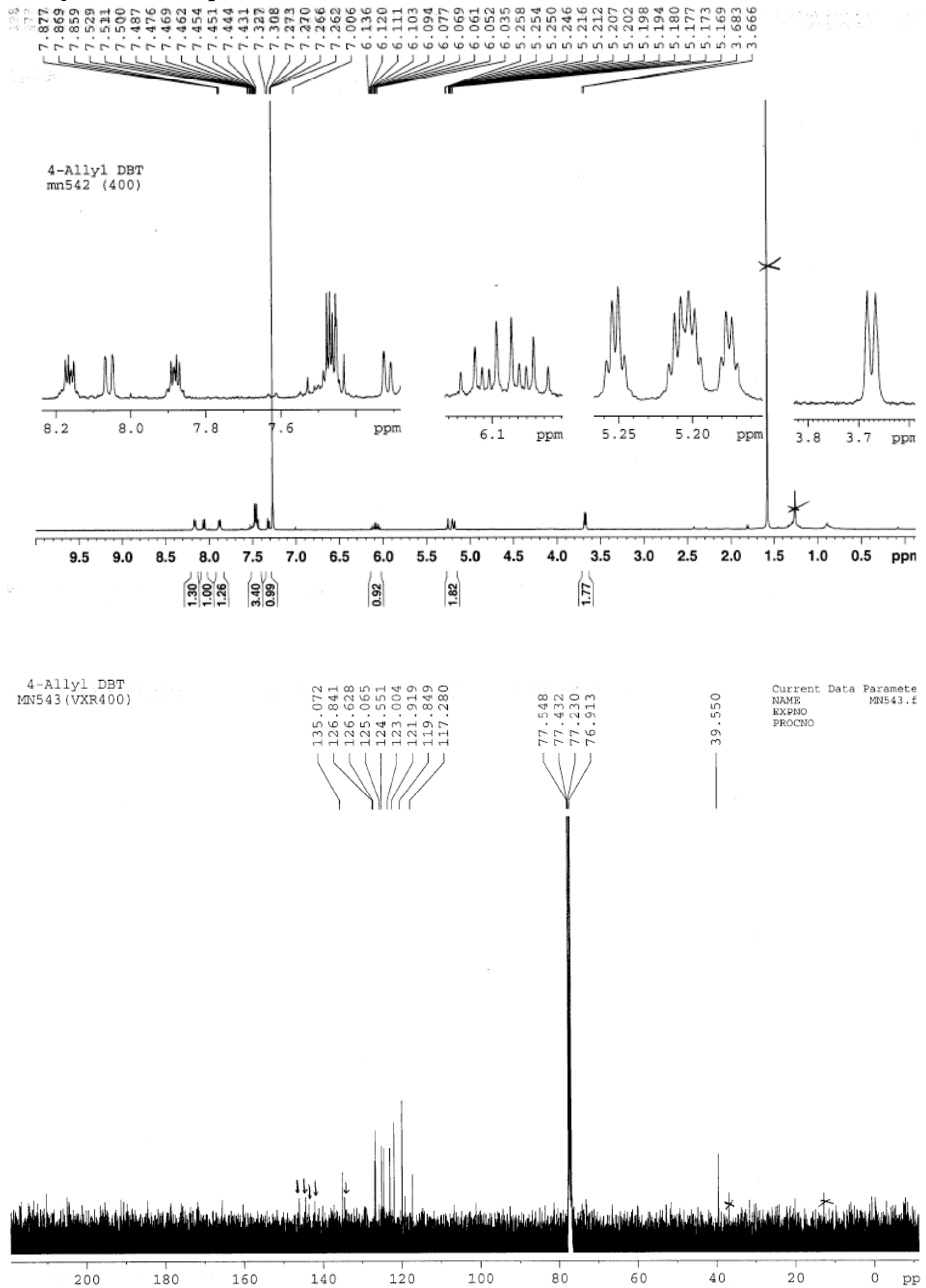


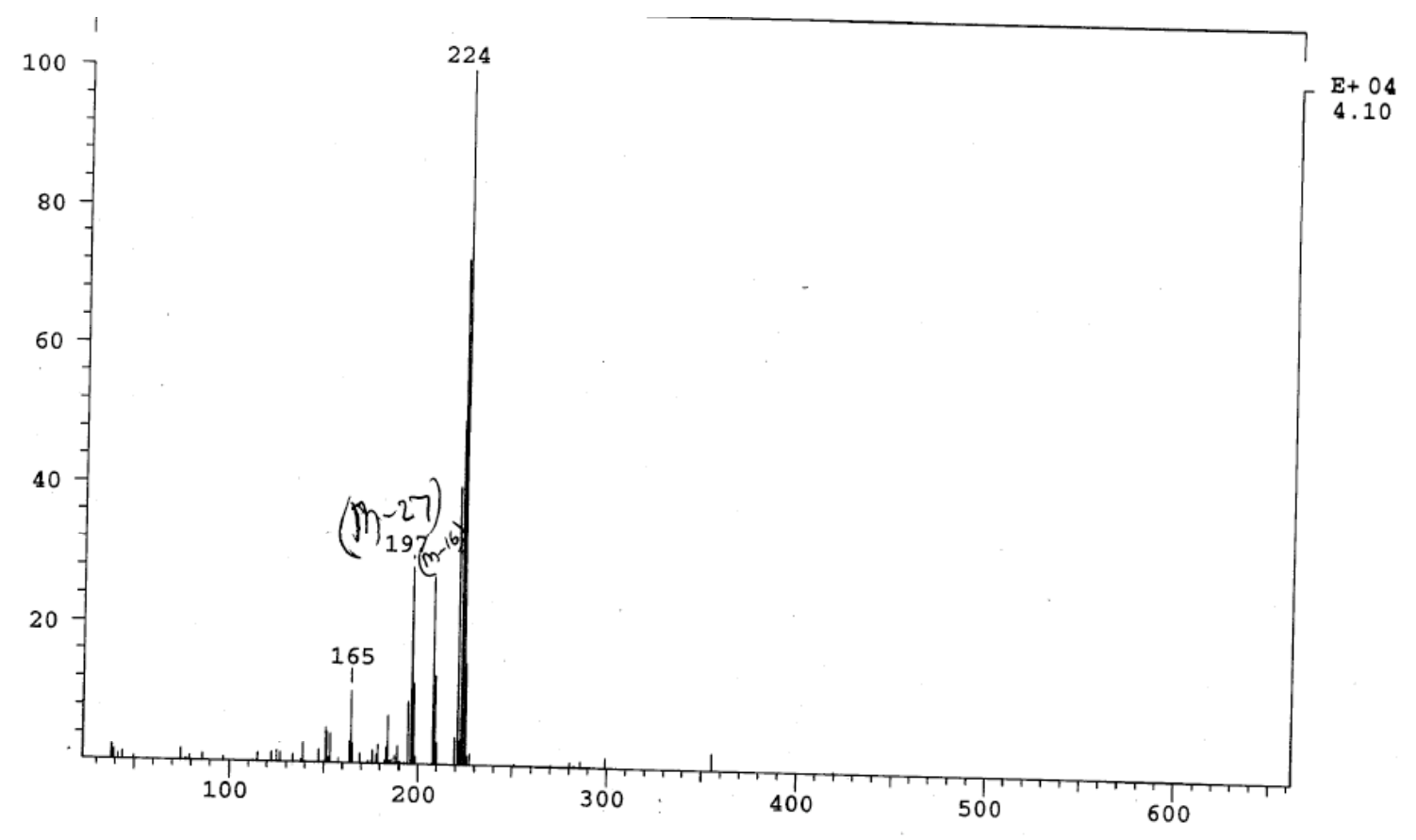




\section{4-(3-Butenyl)dibenzothiophene (4c)}
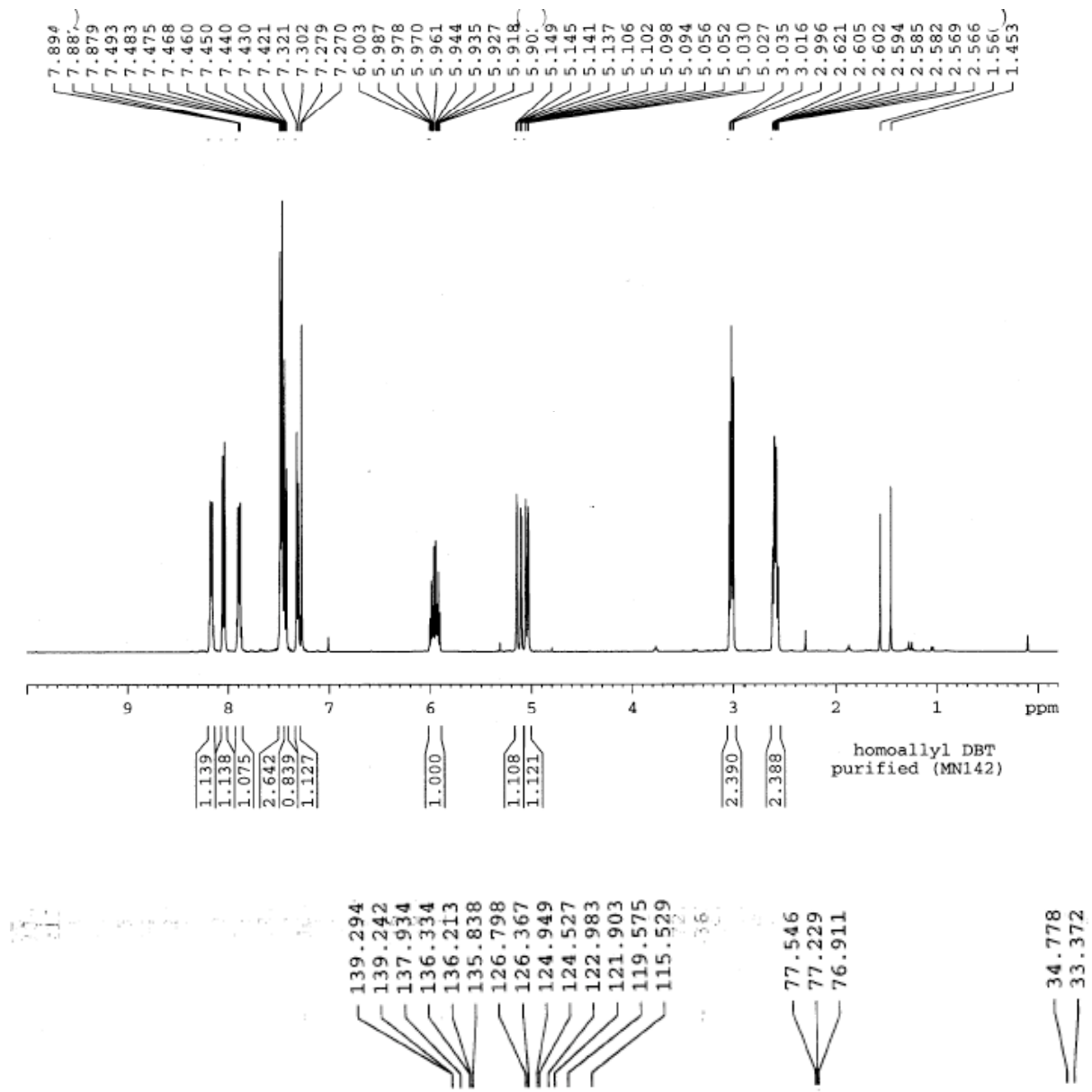

4-Homoallyl DBT

MN542 (400 MHz)

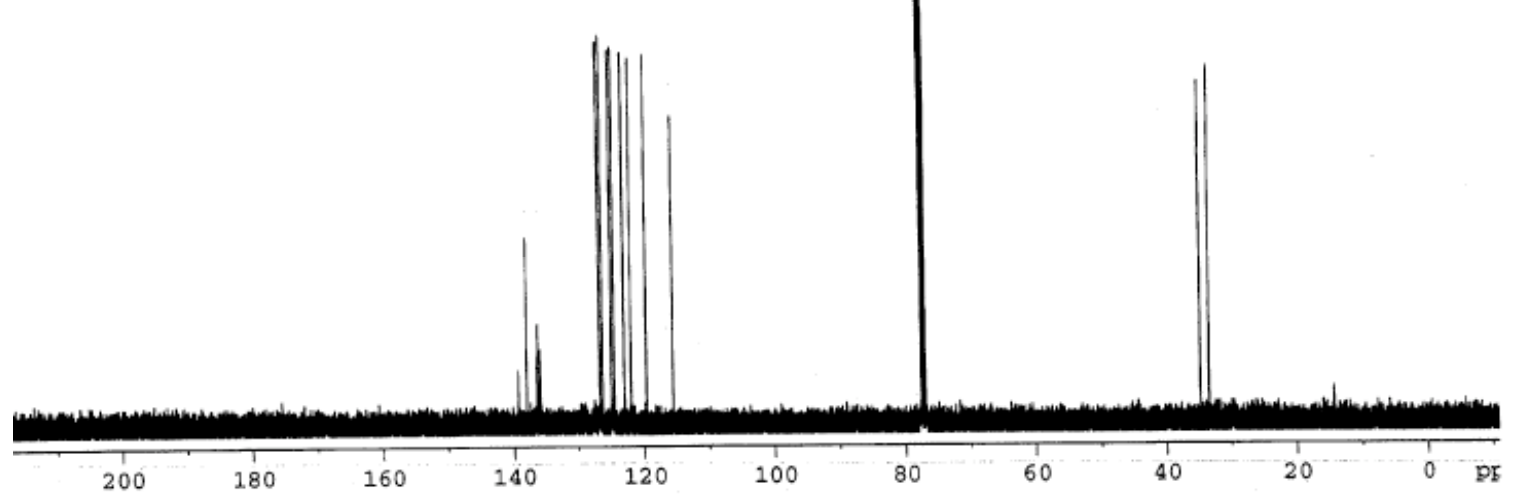




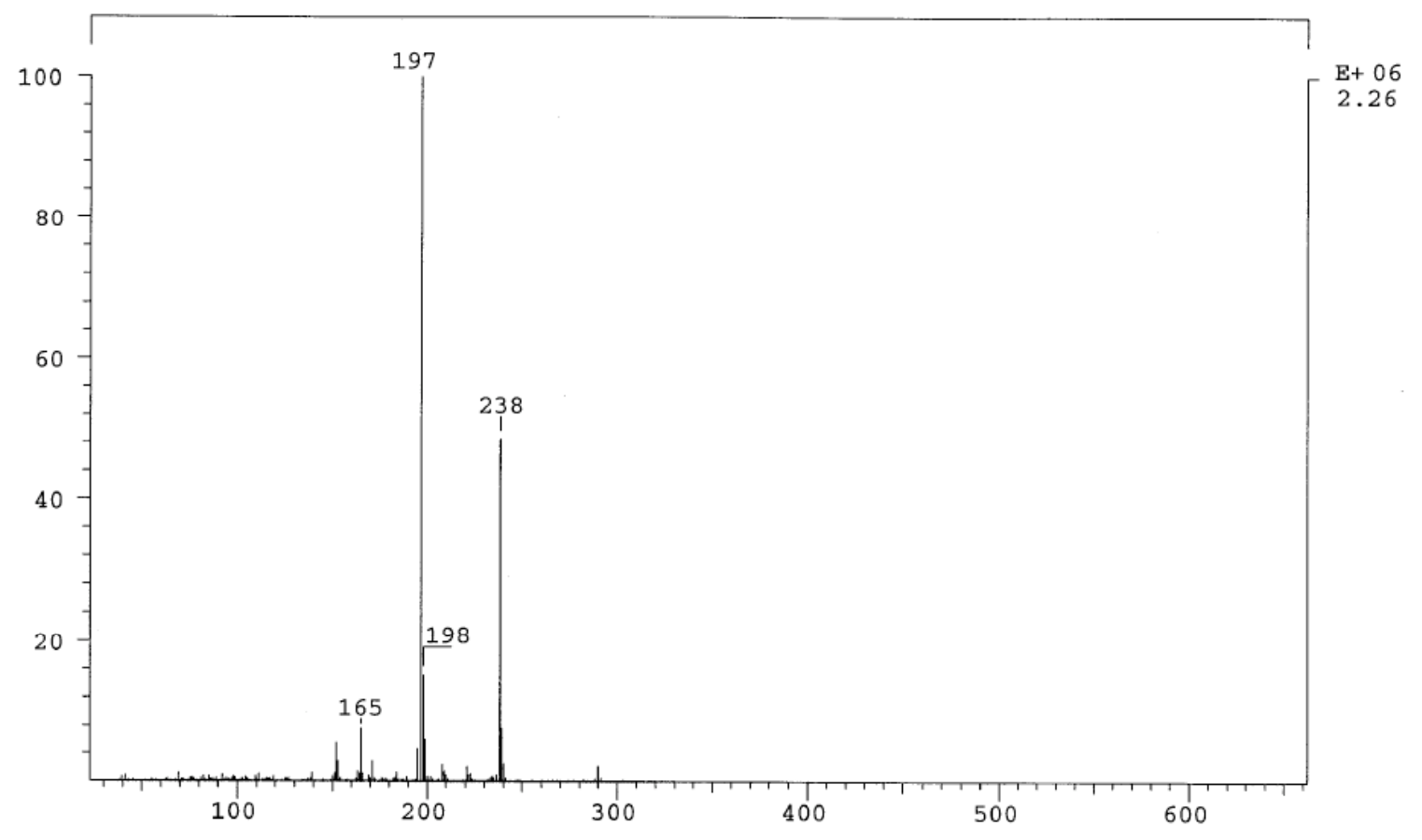




\section{4-Fluorodibenzothiophene}
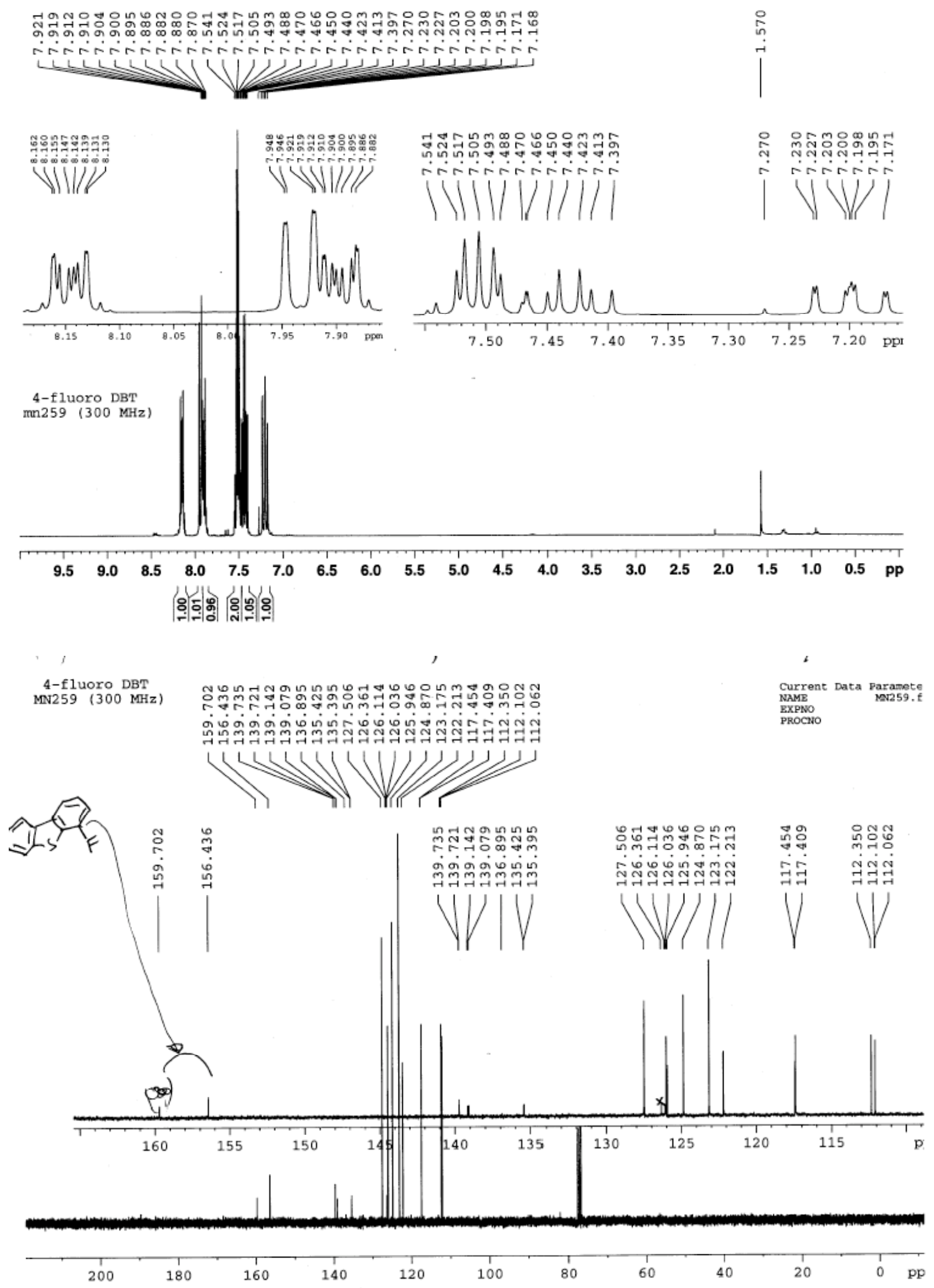


\section{4-Fluorodibenzothiophene oxide (6)}

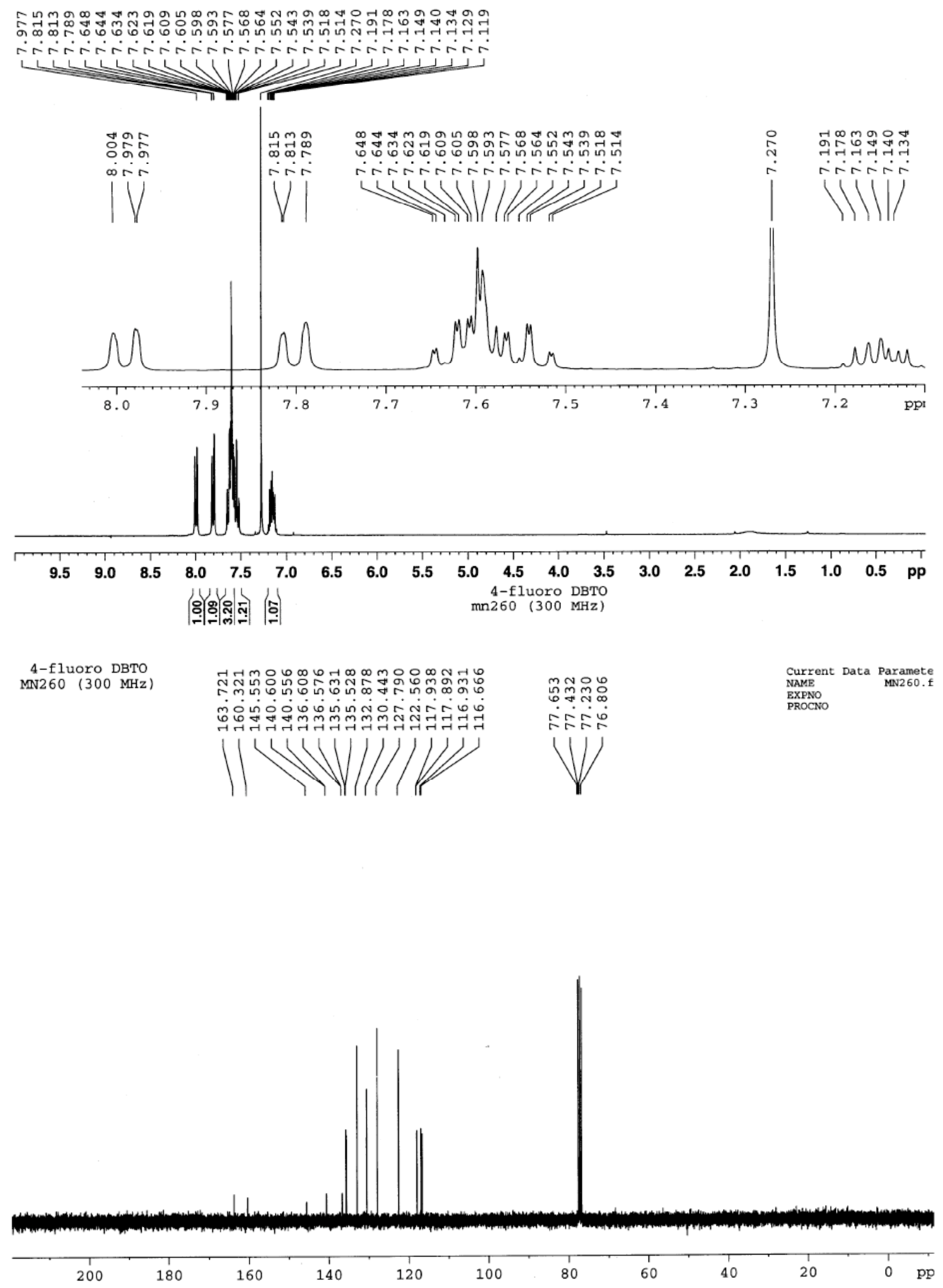




\section{4-methylthiodibenzothiophene (7a)}

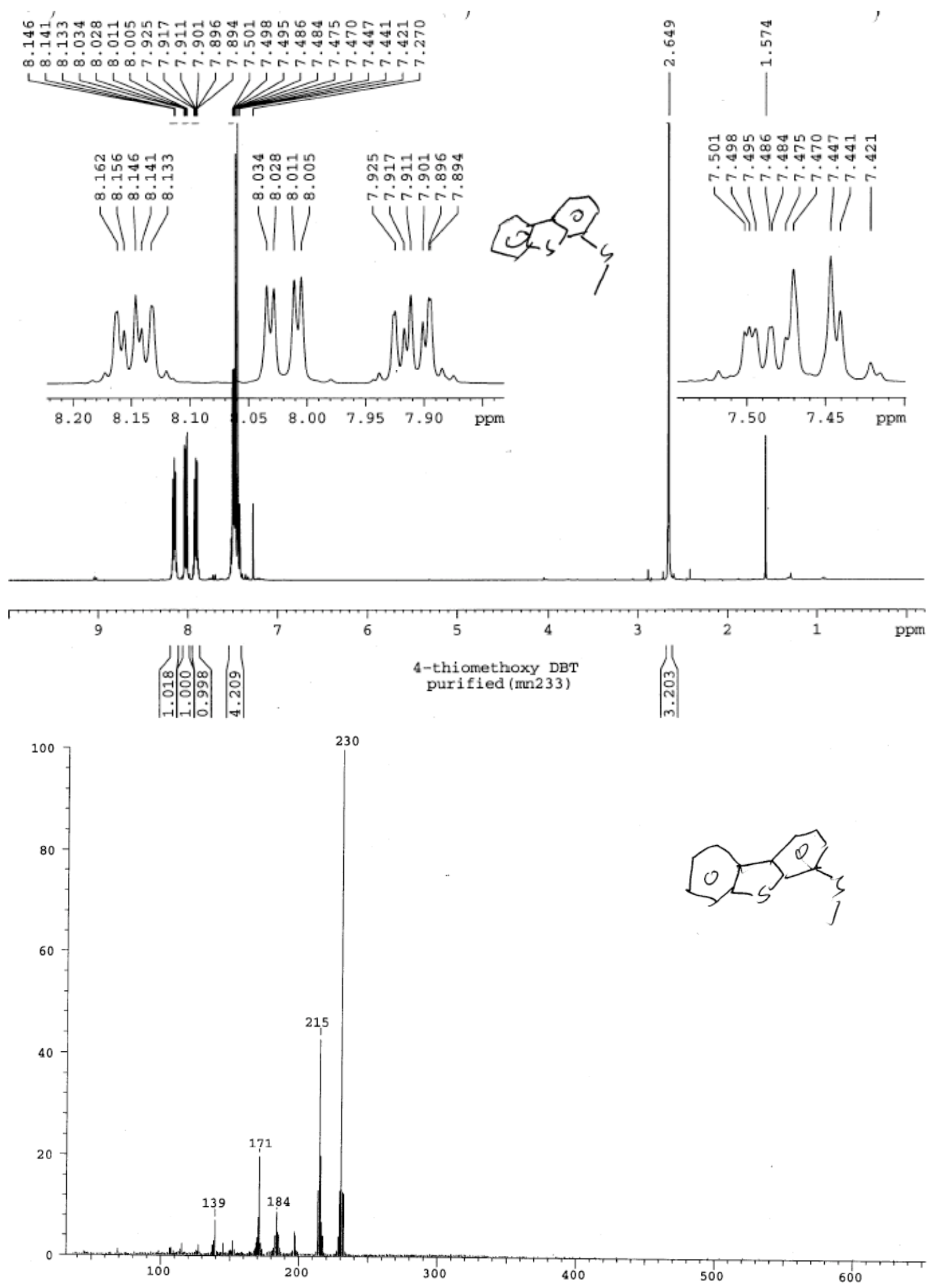


4-(methylthiomethyl)dibenzothiophene (7b)

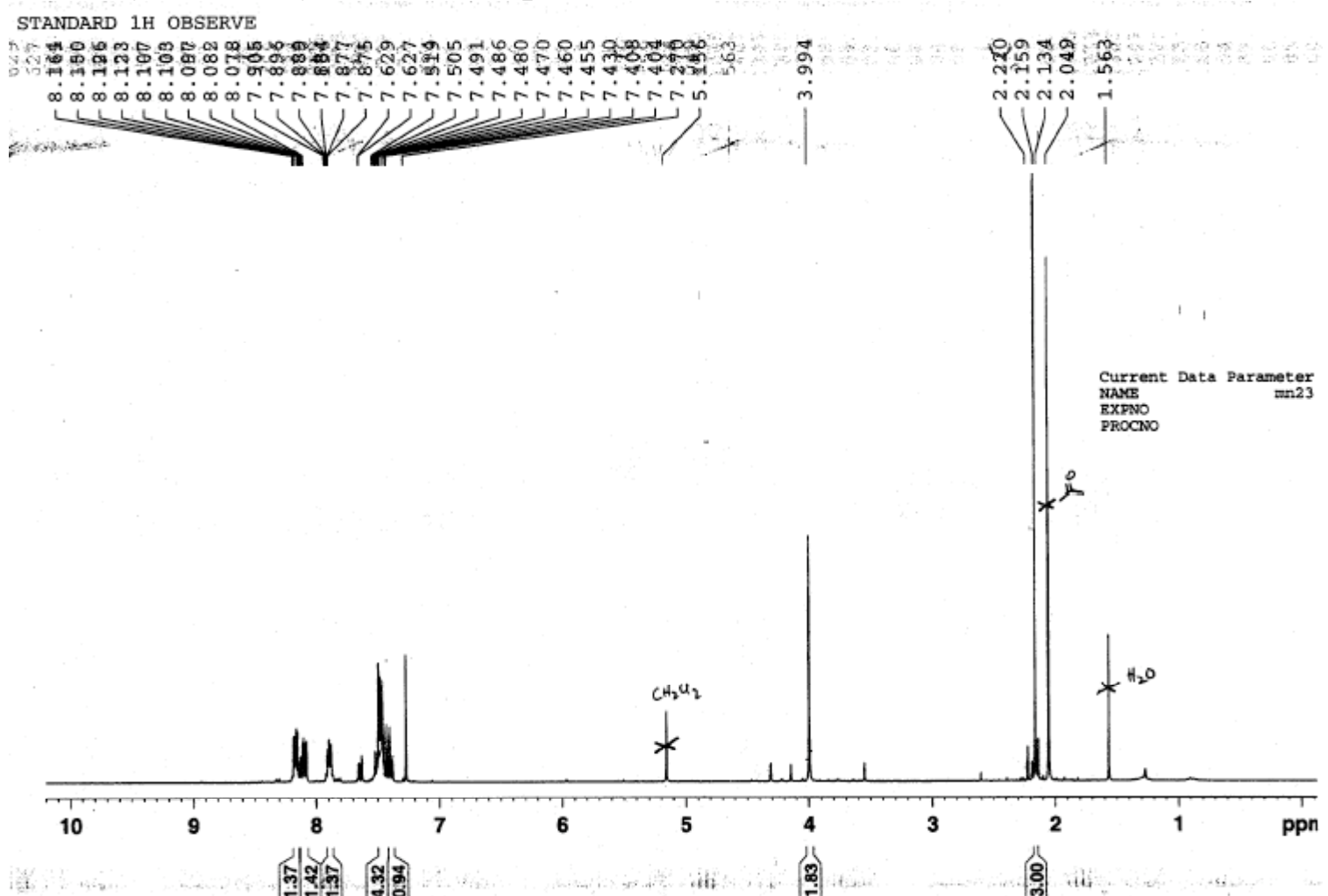

4-Methylthiomethyl
DBT MN551 (400MHz)
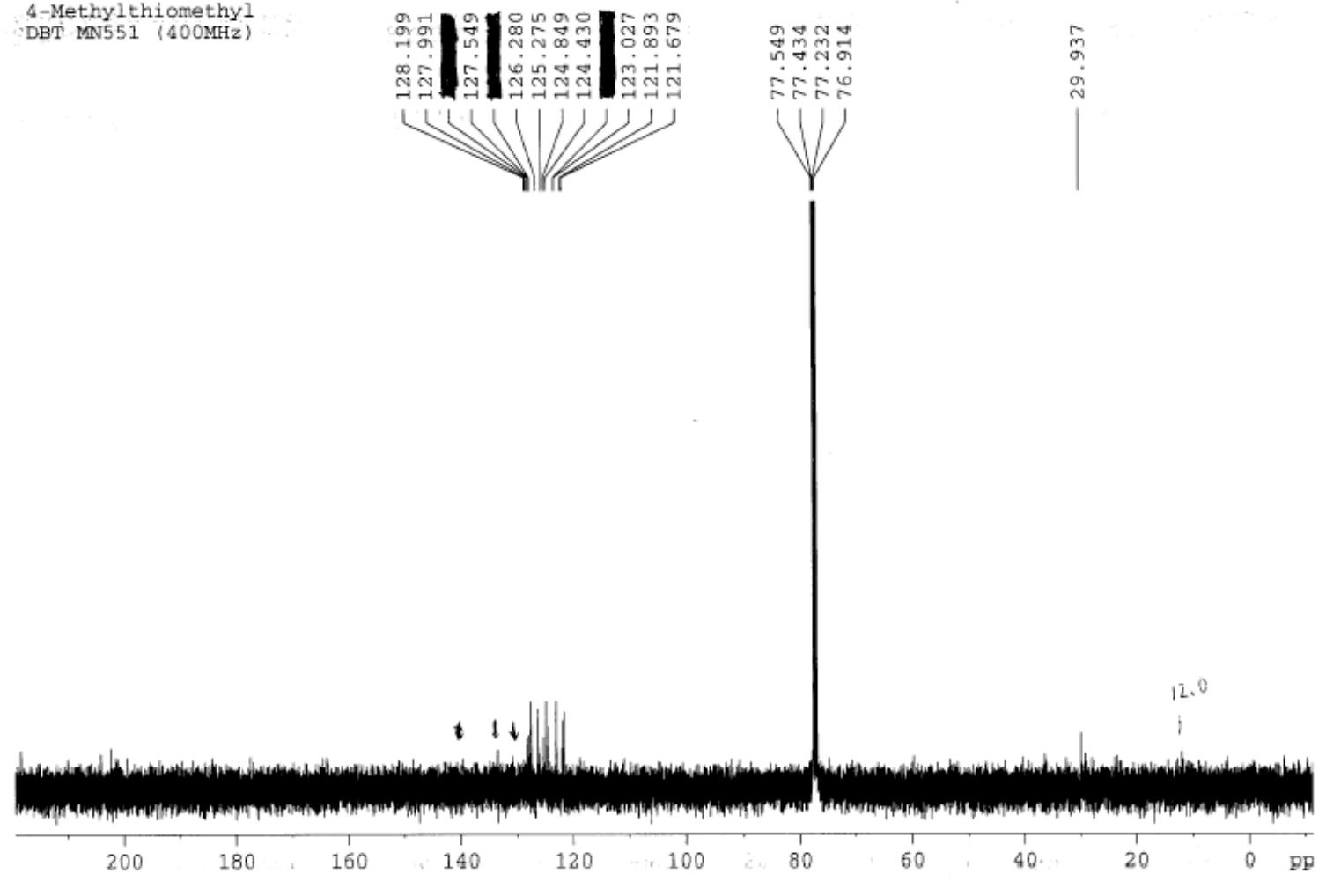


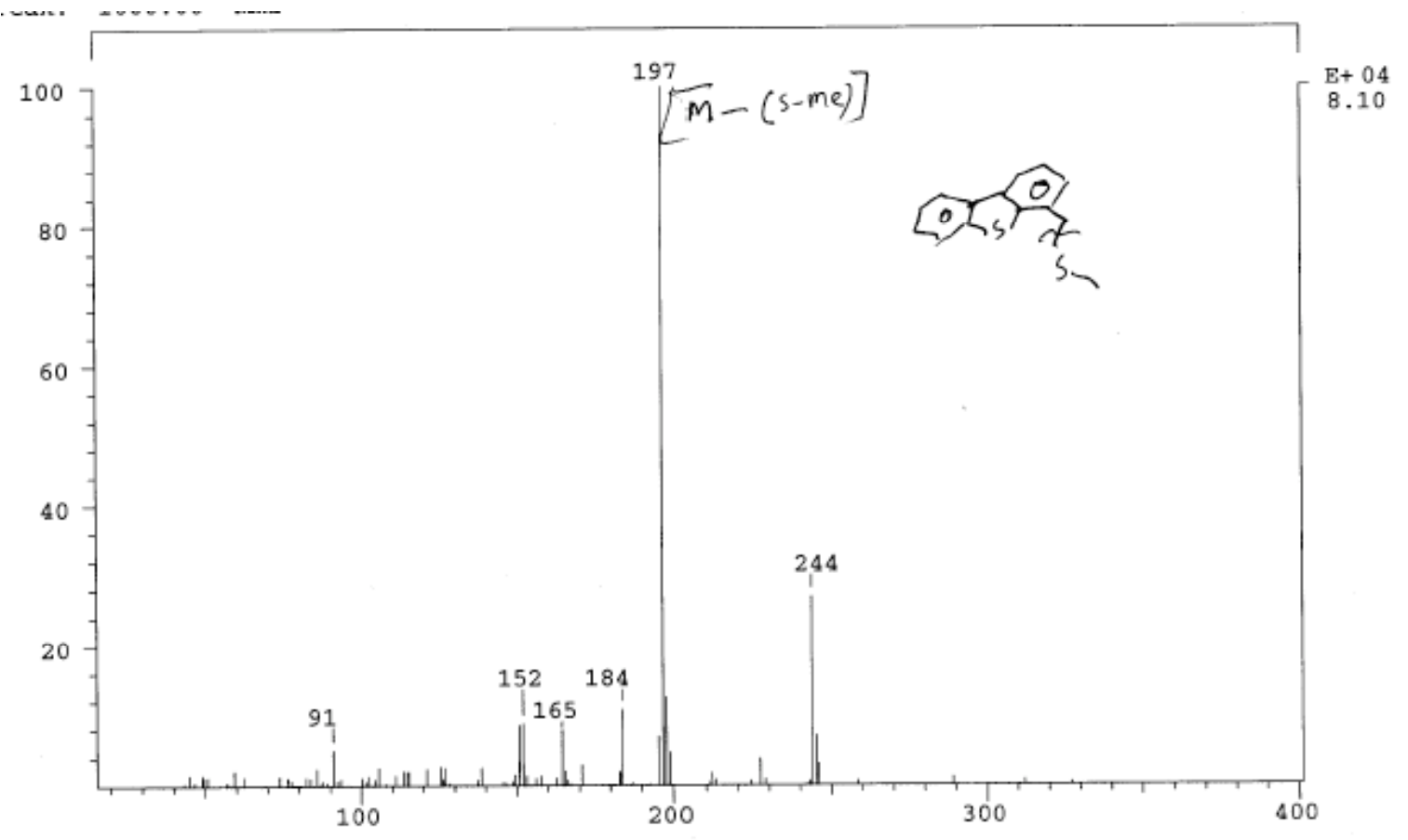




\section{4-(2-Propylthioethyl)dibenzothiophene (7c)}
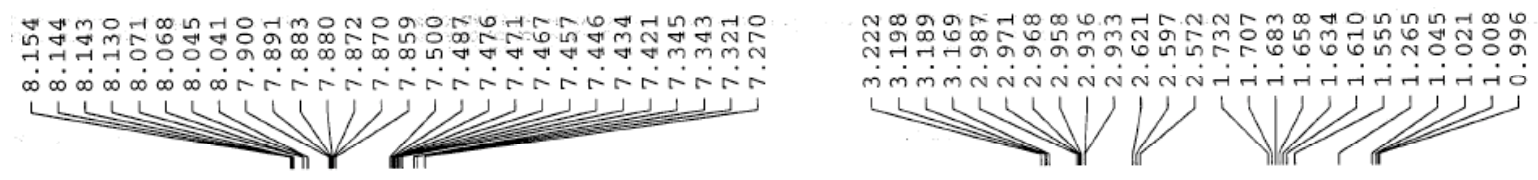

$$
\begin{gathered}
\text { 4-(2-sulfanpropyl) } \\
\text { ethyl DBT mn546 } \\
300 \mathrm{MHz}
\end{gathered}
$$
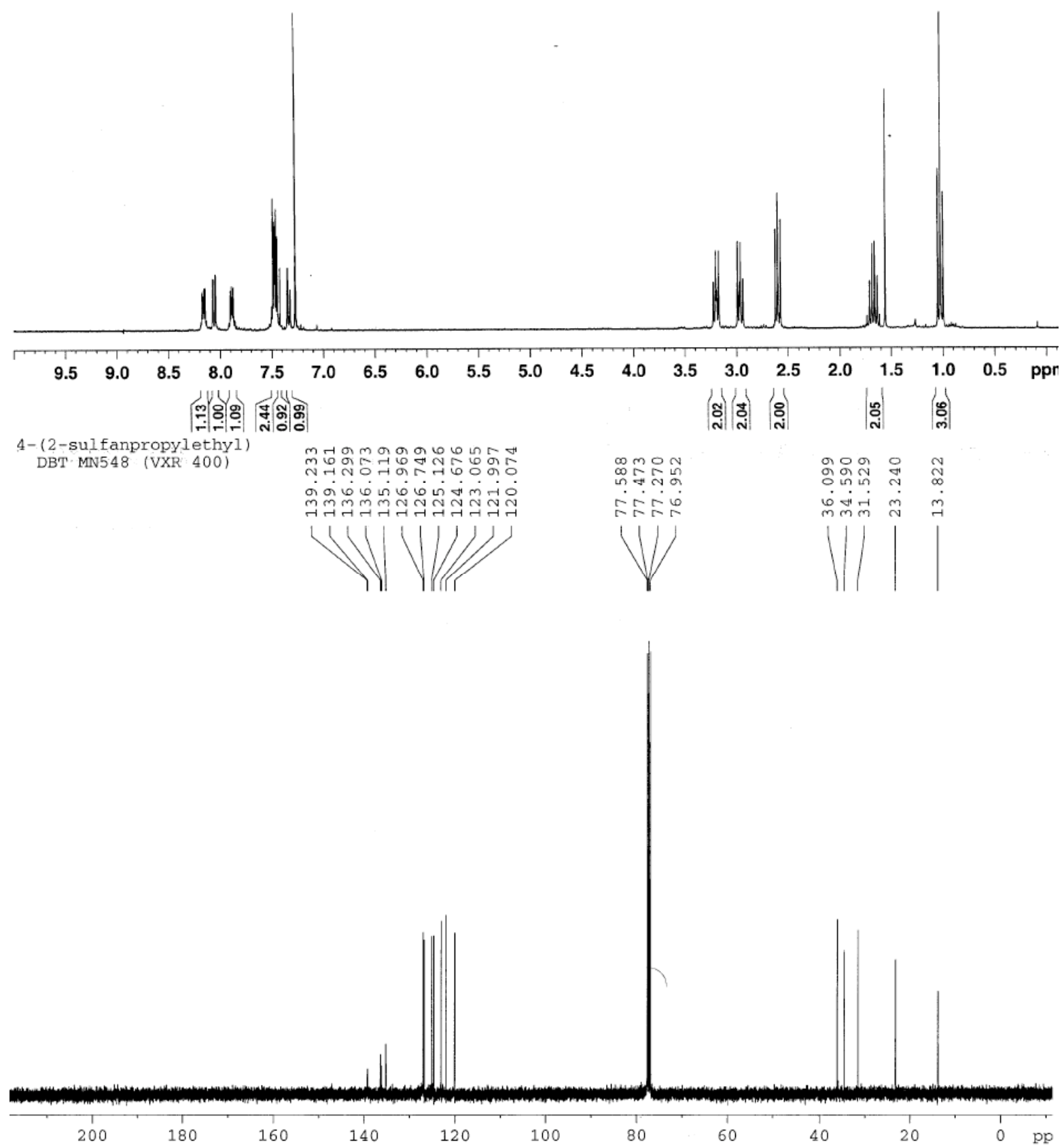


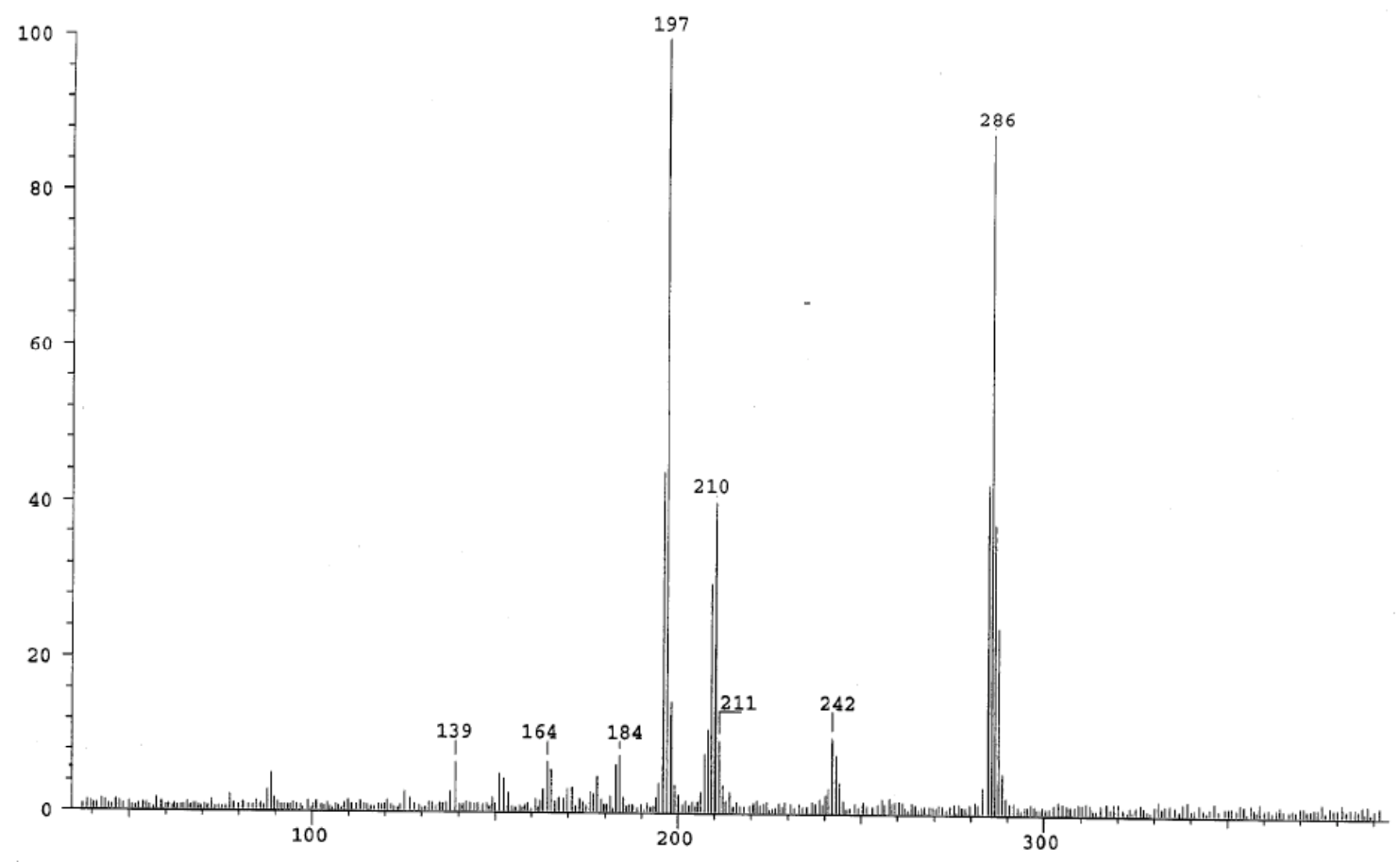

Jate: Wed Oct 20 10:27:57 2004 ICIS: 8.3.0 SP2 for OSF1 (V4.0) build 98-238 from 26-Aug-98 


\section{4-methylsulfinyldibenzothiophene (11a)}
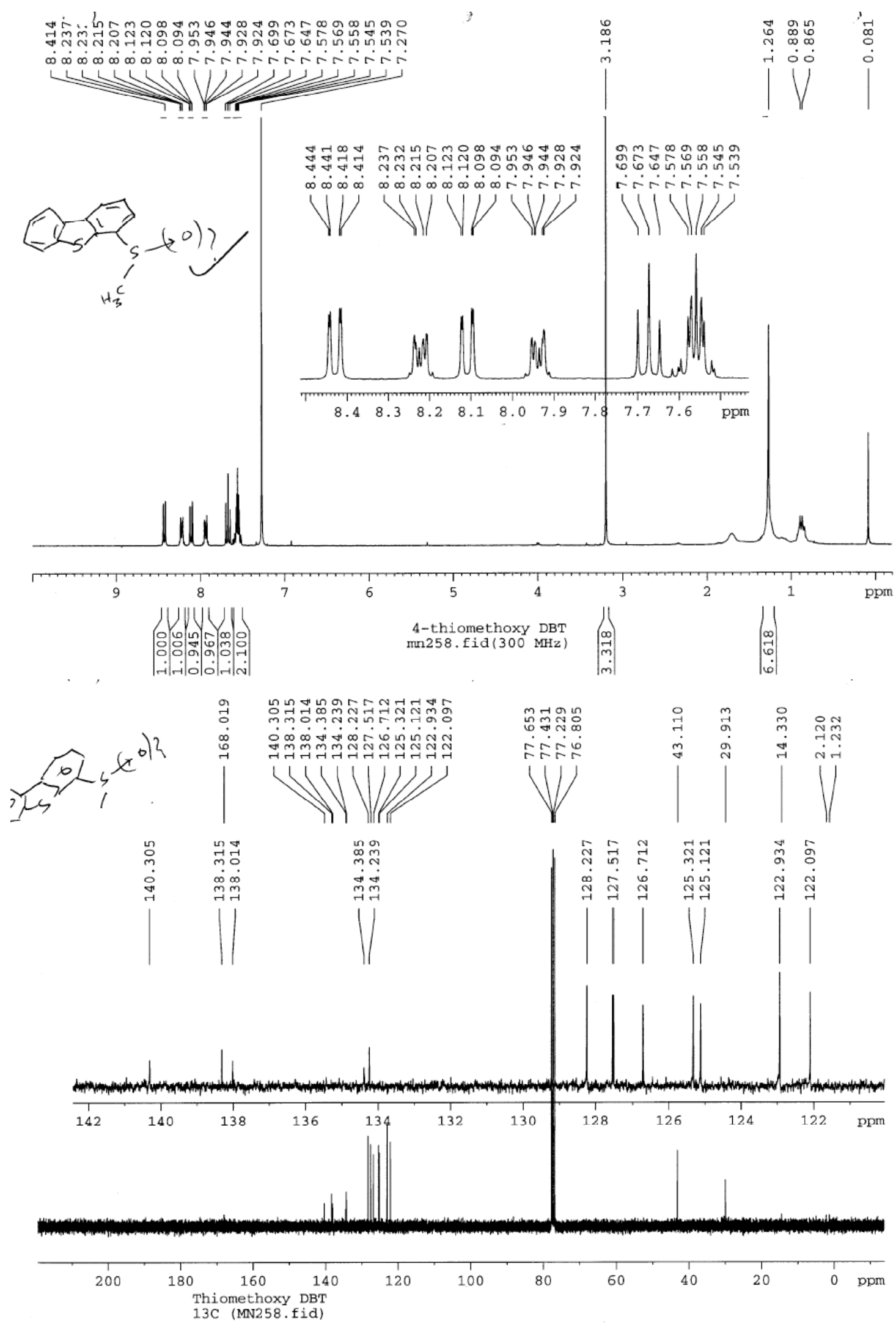


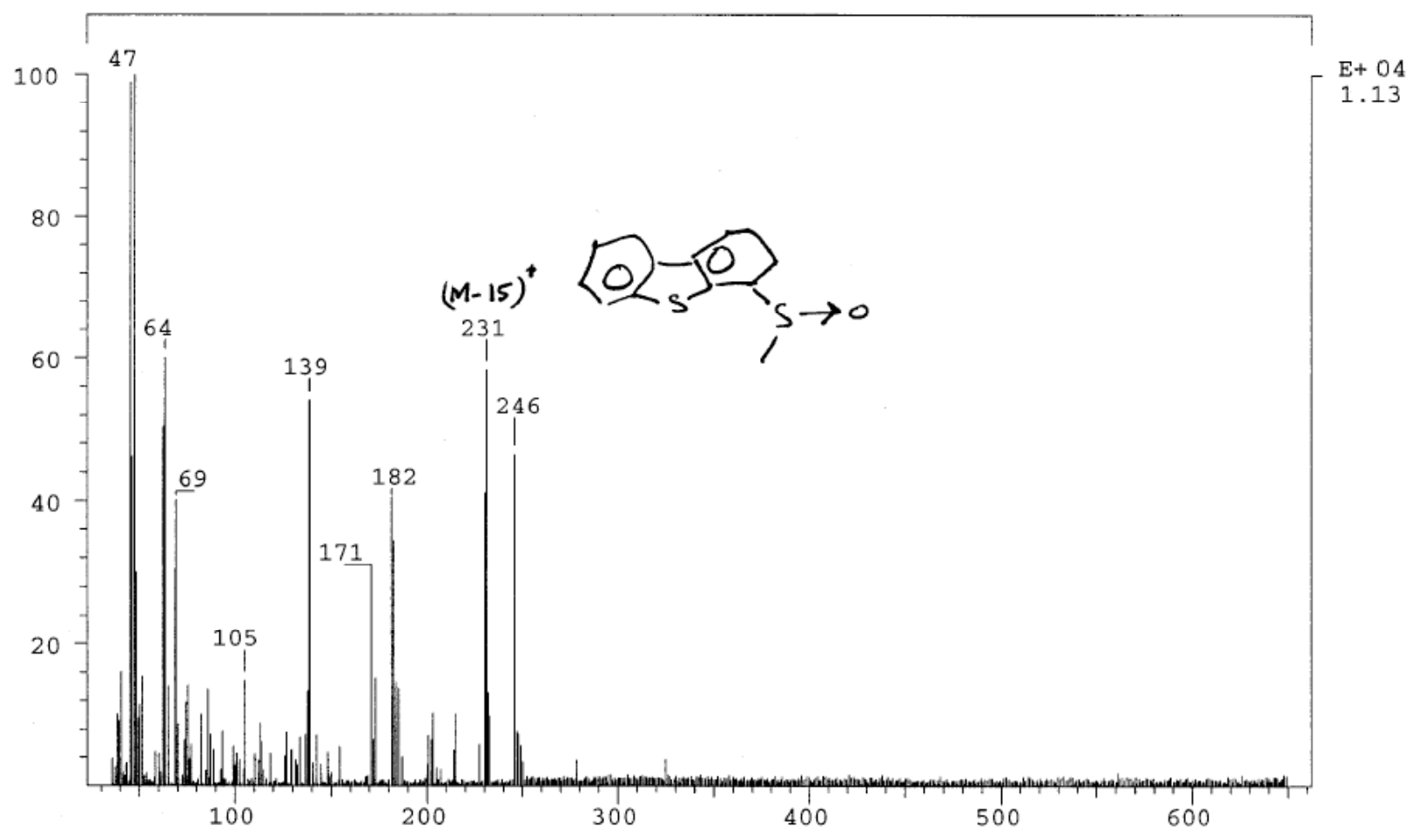




\section{4-(Bromomethyl)dibenzothiophene oxide}

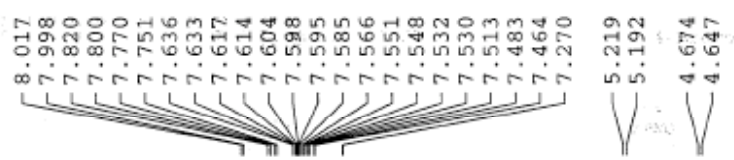

$$
\begin{aligned}
& \text { 4-Bromomethyl DBTO } \\
& 400 \mathrm{MHz} \\
& \begin{array}{l}
\text { Current Data Parameters } \\
\text { NDME } \\
\text { mn543.fid }
\end{array}
\end{aligned}
$$
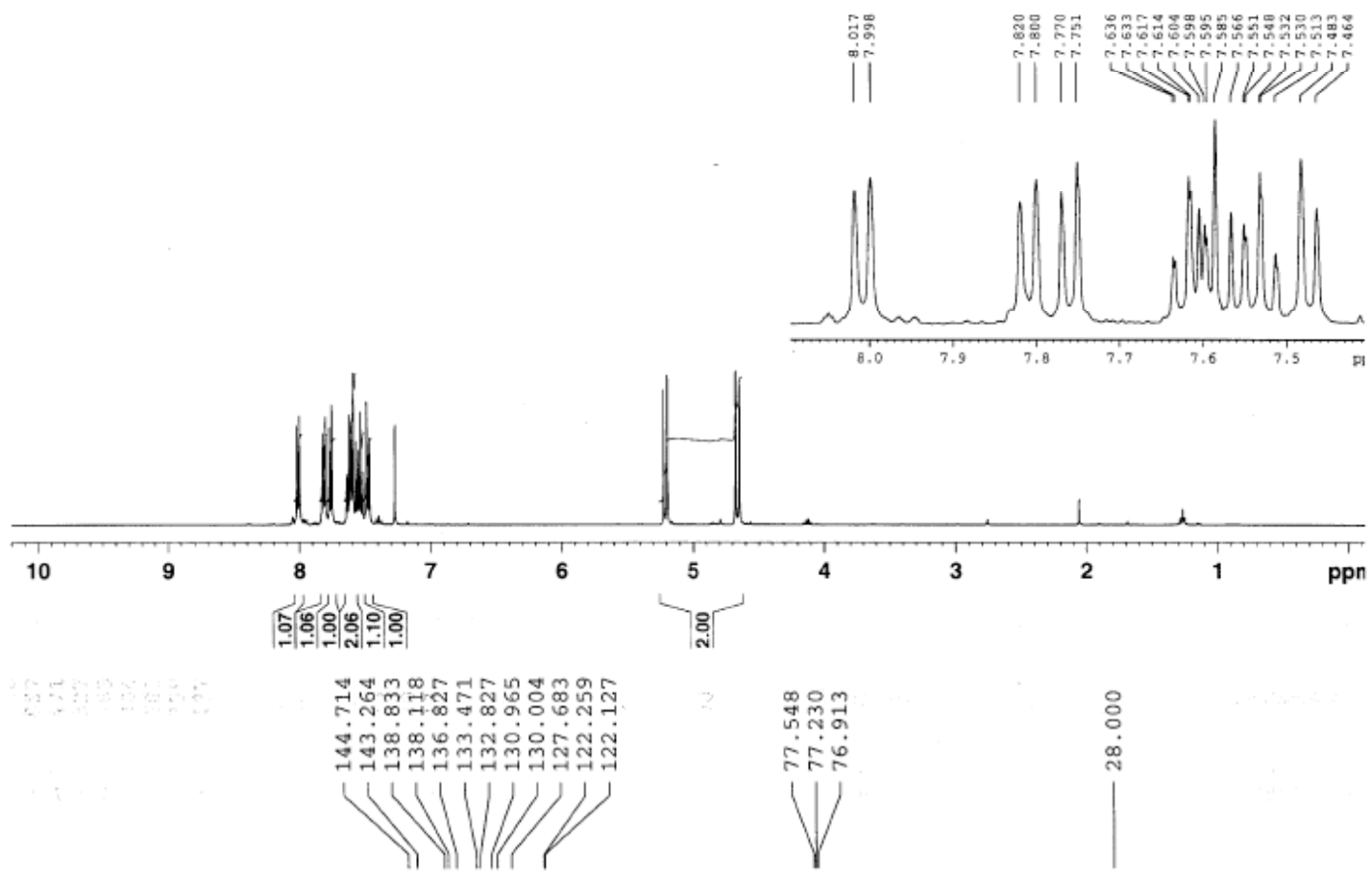

4-Bromonethyl Dand
400 $\mathrm{MHz}$

Current Data Parameters

Mas544,fid

EXPNO

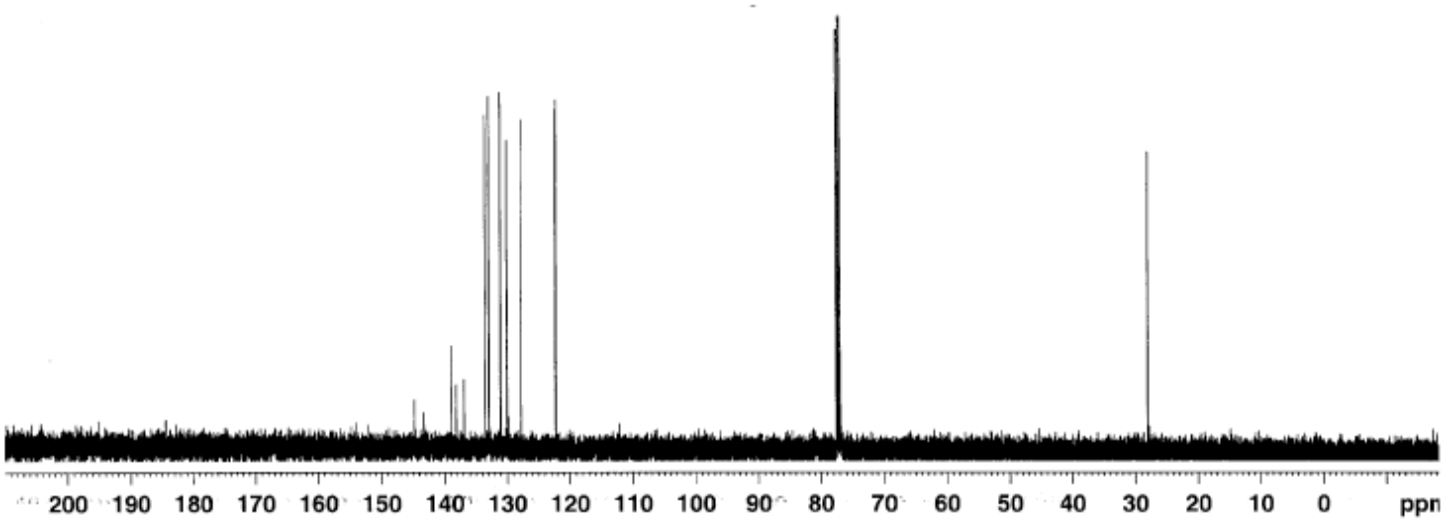




\section{References}

(1) Katritzky, A. R.; Perumal, S. J. Heterocycl. Chem. 1990, 27, 1737-40.

(2) Katritzky, A. R.; Perumal, S. Mag. Reson. Chem. 1990, 28, 914-17.

(3) Snieckus, V.; Beaulieu, F.; Mohri, K.; Han, W.; Murphy, C. K.; Davis, F. A.

Tetrahedron. Lett. 1994, 35, 3465-3468.

(4) Kudo, H.; Tedjamulia, M. L.; Castle, R. N.; Lee, M. L. J. Heterocycl. Chem. 1984, 21, 185-192.

(5) Dunkerton, L. V.; Barot, B. C.; Nigam, A. J. Heterocycl. Chem. 1987, 24, 749-55. 